\title{
Genome-wide transcriptomic analysis of a superior biomass-degrading strain of $A$. fumigatus revealed active lignocellulose- degrading genes
}

Youzhi Miao', Dongyang Liu', Guangqi Li', Pan Li ${ }^{1}$, Yangchun Xu', Qirong Shen ${ }^{1}$ and Ruifu Zhang ${ }^{1,2^{*}}$

\begin{abstract}
Background: Various saprotrophic microorganisms, especially filamentous fungi, can efficiently degrade lignocellulose that is one of the most abundant natural materials on earth. It consists of complex carbohydrates and aromatic polymers found in the plant cell wall and thus in plant debris. Aspergillus fumigatus Z5 was isolated from compost heaps and showed highly efficient plant biomass-degradation capability.

Results: The 29-million base-pair genome of Z5 was sequenced and 9540 protein-coding genes were predicted and annotated. Genome analysis revealed an impressive array of genes encoding cellulases, hemicellulases and pectinases involved in lignocellulosic biomass degradation. Transcriptional responses of A. fumigatus Z5 induced by sucrose, oat spelt xylan, Avicel PH-101 and rice straw were compared. There were 444, 1711 and 1386 significantly differently expressed genes in xylan, cellulose and rice straw, respectively, when compared to sucrose as a control condition.

Conclusions: Combined analysis of the genomic and transcriptomic data provides a comprehensive understanding of the responding mechanisms to the most abundant natural polysaccharides in A. fumigatus. This study provides a basis for further analysis of genes shown to be highly induced in the presence of polysaccharide substrates and also the information which could prove useful for biomass degradation and heterologous protein expression.
\end{abstract}

Keywords: Plant biomass, Lignocellulose, Aspergillus fumigatus, WGS, Transcriptomics

\section{Background}

Plant biomass is the most abundant natural material on earth and the only foreseeable sustainable source of fuels and materials available to humanity [1, 2]. It mainly consists of cellulose, hemicellulose, lignin, pectin and other polymers in ratios that vary between plant species [3]. Cellulose, the main polymeric component of plant biomass [4], pt?>consists of D-glucoside residues linked by beta1,4-glycosidic bonds to form linear polymeric chains of over 10000 glucose residues. Cellulose usually contains regions that are highly crystalline in nature [5]. The

\footnotetext{
* Correspondence: rfzhang@njau.edu.cn

'Jiangsu Key Lab and Engineering Center for Solid Organic Waste Utilization, National Engineering Research Center for Organic-based Fertilizers, Nanjing Agricultural University, Nanjing 210095, P.R. China

${ }^{2}$ Key Laboratory of Microbial Resources Collection and Preservation, Ministry of Agriculture, Institute of Agricultural Resources and Regional Planning, Chinese Academy of Agricultural Sciences, Beijing 100081, P.R. China
}

hydrolysis of cellulose and the degradation of the other polysaccharides in plant biomass require many types of enzymes (e.g., endoglucanase, $\beta$-glucosidase, cellobiohydrolase, endo- $\beta$-1,4-xylanase, $\beta$-xylosidase and numerous other associated enzymes) that work in concert [6]. Even when enzymes work synergistically, degradation of recalcitrant lignocellulose is more difficult than degradation of many other natural materials, such as starch [3].

The key step for the utilization of plant biomass (such as agricultural crop residues, grasses, wood and municipal solid waste) is to degrade it into sugars or oligosaccharides by means of various hydrolytic enzymes. The central technological impediment is the general absence of low-cost technology for overcoming the recalcitrance of lignocellulosic biomass, as the costs of cellulases and hemicellulases contribute substantially to the price $[7,8]$. Studies aimed at understanding and increasing the 
efficiency and productivity of plant biomass-degrading enzymes are at the forefront of this research. By combining enzyme engineering methods (such as mutagenesis, DNA shuffling and protein recombination) with effective screening protocols [9], fold-increased activity and thermostability of enzymes have been achieved [10-12]. However, protein engineering strategies are not always effective and may only make relatively small changes to existing enzymes. Consequently, screening of high-efficiency strains and gene resources is critical for improving biomass utilization [9].

Filamentous fungi are widely used for their capacity to produce extracellular proteins in large quantities [13, 14]. As a result of their saprotrophic lifestyle, filamentous fungi can secret a wide range of enzymes into their habitats, enabling them to metabolize a variety of plant polysaccharides. One filamentous fungus, Neurospora crassa, has long served as a model fungus $[15,16]$ and the molecular techniques developed for $N$. crassa studies have also been widely applied to other filamentous fungi [17]. Meanwhile, Aspergillus genus species (including $A$. niger, $A$. nidulans and $A$. oryzae) have long been used in the enzymatic industry [18, 19]. Another industrial fungus, Trichoderma reese $i$, was reported to have the ability to produce 100 $\mathrm{g}$ of extracellular cellulases per liter [20]. Whole genomes of all of these fungi have been sequenced, which has greatly facilitated the understanding of their highly efficient biomass-degradation mechanisms [21, 22], the design of enzyme cocktails $[5,23]$, and the engineering of strains for higher protein expression.

As we know, A. fumigatus is as versatile in nature as other model fungi. However, there are few detailed studies of its ability to degrade plant biomass, even though some strains of this species are recognized as potent producers of lignocellulolytic and hemicellulolytic enzymes [24, 25]. In our previous study A. fumigatus Z5 isolated from compost heaps of plant straw, has been shown to produce highly thermostable lignocellulosic enzyme activities [26]. Many of the glycoside hydrolases are closely related and have redundant (or partially overlapping) functions, which may facilitate the survival of $A$. fumigatus Z5 under different environmental conditions (e.g., variations in $\mathrm{pH}$, temperature, ionic strength) or may be necessary to effectively depolymerize complex carbohydrate polymers. In order to further understand the biomassdegradation mechanism used by this fungus and to facilitate the development of its applications, the genome sequence of $A$. fumigatus Z5 was determined and the transcriptomic profiles induced by sucrose, xylan, cellulose and rice straw were compared. Combined analysis of the genomic and transcriptomic data provides a comprehensive understanding of the responding mechanisms to the most abundant natural polysaccharides in A. fumigatus, as well as a roadmap for biomass utilization and the industrial application of gene resources or as a host for protein expression.

\section{Results}

General genomic features and comparison with several industrial strains

Phylogenetic analysis (Additional file 1) of strain Z5 and other species in the Aspergillus section Fumigati was based on the $\beta$-tubulin, calmodulin and ITS1 and 2 (rRNA) sequences [27], confirming the attribution of Z5 to $A$. fumigatus. The $A$. fumigatus $\mathrm{Z} 5$ genome was sequenced using the 454 GS FLX platform [28]. A total of 596 contigs were assembled from the reads. Of the contigs, 523 were longer than $2 \mathrm{~kb}$. The N50 has a length of $97 \mathrm{~kb}$ (that is, $50 \%$ of all bases are contained in contigs of at least $97 \mathrm{~kb}$ ). These contigs were assembled into 24 scaffolds with a total length of $29.4 \mathrm{Mb}$ and an N50 length of $2.29 \mathrm{Mb}$. The genome was predicted to contain 9540 genes that encode proteins with a length greater than 80 amino acid residues. The genome was composed of 8 chromosomes, as determined by matching scaffolds with the chromosomes of $A$. fumigatus AF293 [29] (Additional file 2). Strain Z5 has 245 unique proteins when compared to A. fumigatus AF293 and $N$. fischeri NRRL 181, and of these unique proteins, 218 were annotated as hypothetical proteins. Genome statistics

Table 1 Genome features of A. fumigatus Z5

\begin{tabular}{lc}
\hline Features & Values \\
\hline Sizes (bp) & $29,369,860$ \\
Chromosomes & 8 \\
G + C content (\%) & $49.2 \%$ \\
Protein coding genes & 9540 \\
Mean gene length (bp) & 1719 \\
Percent coding & $55.9 \%$ \\
Percent genes with introns & $82.3 \%$ \\
Genes of unknown function & $21.2 \%$ \\
Exons & 3.28 \\
Mean number per gene & 463.5 \\
Mean length (bp) & $54.0 \%$ \\
G + C content (\%) & \\
Introns & 2.28 \\
Mean number per gene & 87.3 \\
Mean length (bp) & $45.5 \%$ \\
G + C content (\%) & \\
Intergenic regions & 1356 \\
Mean length (bp) & $44 \%$ \\
G + C content (\%) & \\
RNA & \\
tRNA & \\
\hline
\end{tabular}


are presented in Table 1. Several Z5 scaffolds matched different chromosomes of A. fumigatus AF293, suggesting that duplication of genomic fragments likely occurred in strain Z5 during the evolution of Z5 from the common ancestor.

A. fumigatus Z5 has 627 significantly different proteincoding genes compared to other 5 well-studied filamentous fungi (Table 2). InterPro [30] identification of conserved domains and families among predicted proteins of A. fumigatus Z5 could provide an overview of the expression capability of this filamentous fungus (Additional files 3 and 4). A large expansion in the InterPro category corresponding to the major facilitator superfamily (MFS) in Aspergillus genus may reflect their powerful ability to transport small solutes compared with 2 other filamentous fungi, T. reesei and N. crassa. About the number of sugar transporters in MFS transporters, Aspergillus genus has an average of 106 sugar transporters, nearly two folds of those in $T$. reesei and $N$. crassa, and this expansion of $\mathrm{Z} 5$ was happened in the sugar porter (SP) family (TC no. 2.A.1.1) which covered $90 \%$ of all the sugar transporters in these fungi (Fig. 1). Consistent with the capacity for efficient lignocellulose degradation, strain Z5, like three other Aspergillus species (A. niger, A. nidulans, A. ory$z a e)$, has a relatively high number of glycoside hydrolase superfamily genes (IPR017853). Compared to T. reesei and N. crassa, the Aspergillus genus has more fungalspecific transcription factors (IPR007219), suggesting that Aspergillus species have more complex or redundant regulation mechanisms. A. fumigatus Z5 has 81 cytochrome P450 genes (IPR001128), which play important roles in secondary metabolism, while A. fumigatus AF293 has only 65 cytochrome P450 genes [31]. In order to identify lignocellulose-degrading proteins, the protein domains encoded by the A. fumigatus Z5 genome were compared with the genomes of six other fungi widely used in industry (Additional file 4). The Aspergillus strains were found to contain more glycoside hydrolases, pectate lyases (pectin esterases) and tannases (feruloyl esterases) than when compared to $T$. reesei and $N$. crassa.

Using the cluster of orthologous groups (COG) classification database [32], all available proteins from six filamentous fungi and a strain of Saccharomyces cerevisiae were clustered into 25 COG functional categories (Fig. 2;

Table 2 Specific proteins in six filamentous fungi

\begin{tabular}{lcc}
\hline Species & Specific proteins & Total proteins \\
\hline A. fumigatus Z5 & 627 & 9540 \\
A. niger & 3664 & 14097 \\
A. nidulans & 1006 & 10680 \\
A. oryzae & 1158 & 12030 \\
N. crassa & 2645 & 9907 \\
T. reesei & 1374 & 9143 \\
\hline
\end{tabular}

see also Additional file 5). There were no differences in any of the information storage and processing categories ( $\mathrm{J}$ to $\mathrm{B}$ ) or in most of the cellular processes and signaling categories ( $\mathrm{D}$ to $\mathrm{O}$ other than $\mathrm{V}$ and $\mathrm{M}$ ), even though there were large differences in genome sizes. Most of the gene family expansions in filamentous fungi, when compared to Saccharomyces cerevisiae, were found in gene families predicted to have roles in defense mechanisms $(\mathrm{V})$, extracellular structures $(\mathrm{M})$ and metabolism ( $\mathrm{C}$ to E, I to Q). Genes in these expanded categories were more abundant in the Aspergillus genus than in N. crassa or T. reesei.

\section{Carbohydrate-active enzymes in A. fumigatus Z5}

Carbohydrate-active enzymes (CAZymes) are categorized into different classes (glycoside hydrolases, glycosyltransferases, polysaccharide lyases, carbohydrate esterases, auxiliary activities and carbohydrate-binding modules) in the CAZy database (http://www.cazy.org) [33]. CAZymes cleave, build and rearrange oligo- and polysaccharides. These functions play important roles in fungi such as $A$. fumigatus and are vital for optimizing biomass degradation by these species [5]. Given the importance of this protein family for biomass utilization, a detailed examination of the CAZome of $A$. fumigatus Z5 was performed.

The genome of $A$. fumigatus Z5, which efficiently degrades plant polysaccharides, encodes approximately 529 putative carbohydrate-active enzymes (280 glycoside hydrolases, 98 glycosyltransferases, 14 polysaccharide lyases, 69 carbohydrate esterases, 58 auxiliary activities and 61 carbohydrate-binding modules), which were divided into at least 115 distinct families (Additional file 6). Compared to several available A. fumigatus and $N$. fisheri genomes in the Aspergillus section Fumigati (Table 3), no significant differences were found in the distribution of CAZyme classes between our strain Z5 and other three $A$. fumigatus, and $92.3 \%$ CAZyme proteins in $N$. fisheri genome showed homogenous with those of $A$. fumigatus. In fact, Aspergillus genus has the leading position in the number of CAZyme genes among the 103 publicly available fungal genomes [34]. Figure 3 depicts the distribution of CAZyme genes by family and the responses of gene families to the induction by extracellular polysaccharides. Of the glycoside hydrolase genes, $39.2 \%$ had significantly higher expression levels (q-value $\leq 0.0001$ and |the ratio of the $R P M$ values $\mid \geq 4$ ) after polysaccharide induction than after sucrose induction (as discussed below). Most of the glycoside hydrolases with significantly increased expression were responsible for the degradation of cellulose, hemicellulose and pectin (Fig. 3). Under the same conditions, the carbohydrate-binding module, carbohydrate esterase, auxiliary activities and polysaccharide lyase families also had a relative high percentage of induced genes (45.9, 39.1, 24.1 and $64.3 \%$ respectively). However, 


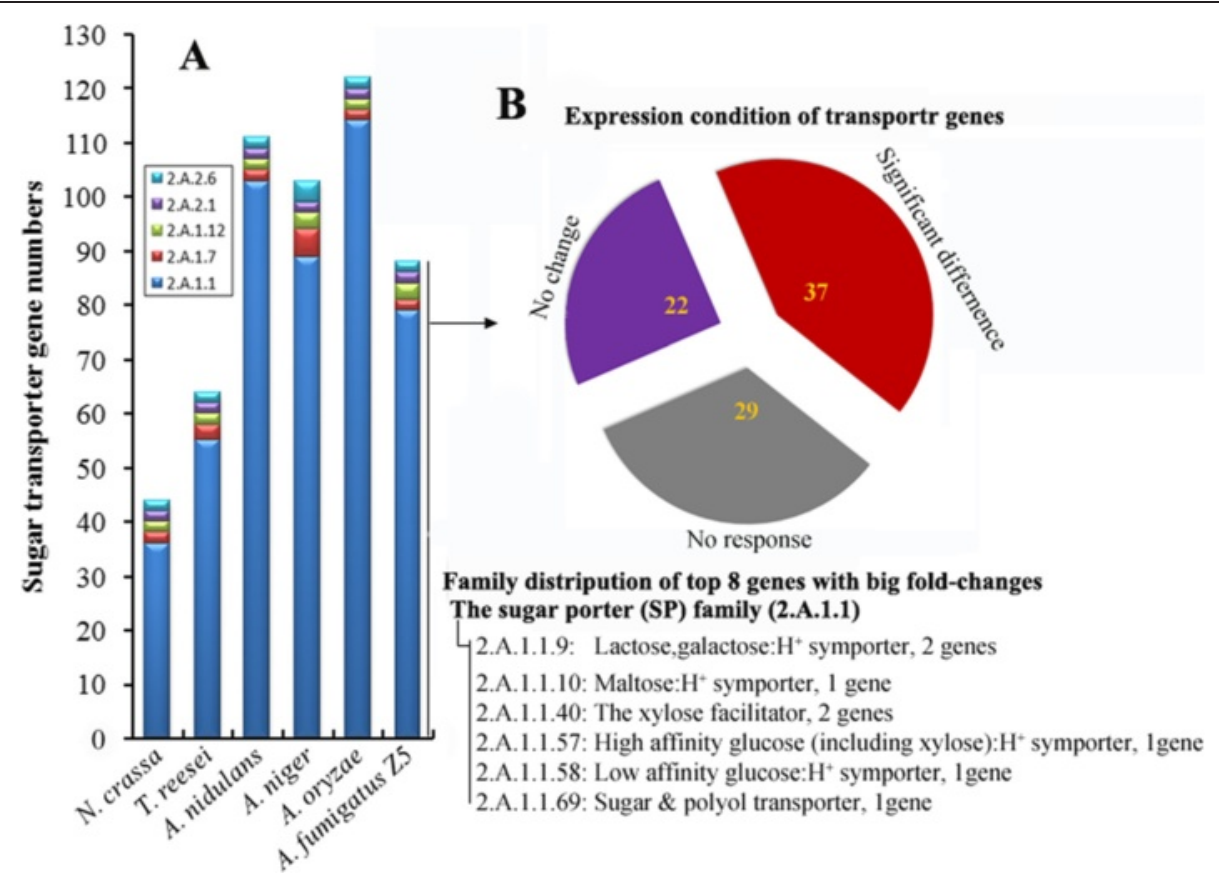

Fig. 1 The sugar transporter genes. a Comparison of the sugar transporter genes in six filamentous fungi. 2.A.1.1, the sugar porter (SP) family; 2.A.1.7, the fucose: $\mathrm{H}^{+}$symporter (FHS) family; 2.A.1.12, the sialate: $\mathrm{H}^{+}$symporter (SHS) family; 2.A.2, the glycoside-pentoside-hexuronide (GPH):cation symporter family. $\mathbf{b}$ Expression conditions of the sugar transporter genes in strain Z5. Red indicated differently expressed genes in polysaccharidesinduced samples when compared to sucrose control, gray represented low expression genes (RPM $<20)$ in all samples including sucrose control and purple represented the considerable expressed genes but no differences among 4 treatments. The exact families were given for these 8 genes which expression changed the most

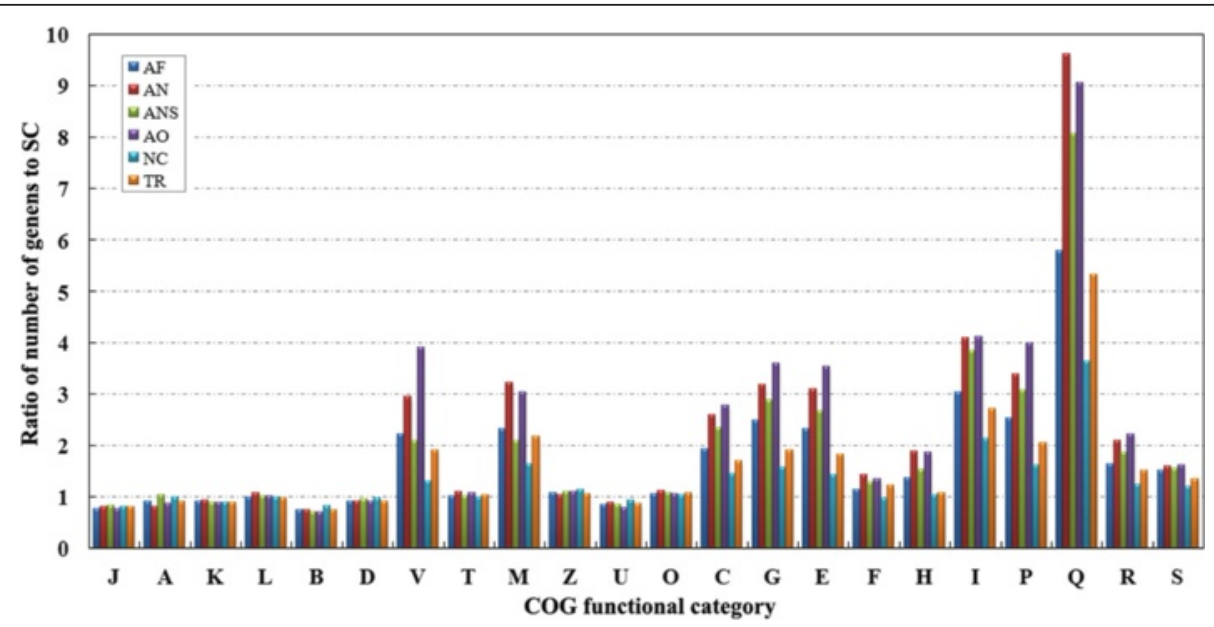

Fig. 2 Comparison of relative gene numbers in each COG. The ratios of gene counts in A. fumigatus Z5 (AF), A. niger (AN), A. nidulans (ANS), A. oryzae (AO), N. crassa (NC) and T. reesei (TR) to gene counts in S. cerevisiae (SC) in each COG category were calculated. COGs with a gene number $\leq 5$ for each species $(\mathrm{Y}, \mathrm{N}$ and $\mathrm{W}$ ) are not displayed to avoid misinterpretation resulted from potentially low reliability. J, Translation, ribosomal structure and biogenesis; A, RNA processing and modification; K, Transcription; L, Replication, recombination and repair; B, Chromatin structure and dynamics; D, Cell cycle control, cell division, chromosome partitioning; Y, Nuclear structure; V, Defense mechanisms; T, Signal transduction mechanisms; M, Cell wall/membrane/envelope biogenesis; N, Cell motility; Z, Cytoskeleton; W, Extracellular structures; U, Intracellular trafficking, secretion, and vesicular transport; O, Posttranslational modification, protein turnover, chaperones; C, Energy production and conversion; G, Carbohydrate transport and metabolism; E, Amino acid transport and metabolism; F, Nucleotide transport and metabolism; H, Coenzyme transport and metabolism;l, Lipid transport and metabolism; P, Inorganic ion transport and metabolism; Q, Secondary metabolites biosynthesis, transport and catabolism; R, General function prediction only; S, Function unknown 
Table 3 CAZyme gene numbers in A. fumigatus and N. fischeri

\begin{tabular}{|c|c|c|c|c|c|c|c|c|c|c|c|c|c|c|c|c|c|c|c|}
\hline \multirow[t]{2}{*}{ Species } & \multirow{2}{*}{$\begin{array}{l}\text { CAZyme } \\
\text { proteins }\end{array}$} & \multicolumn{3}{|l|}{$\mathrm{GH}$} & \multicolumn{3}{|l|}{ GT } & \multicolumn{3}{|l|}{ CE } & \multicolumn{3}{|l|}{$\mathrm{PL}$} & \multicolumn{3}{|c|}{ CBM } & \multicolumn{3}{|l|}{ AA } \\
\hline & & $\mathrm{N}$. & $\mathrm{H}$. & $\mathrm{F}$. & $\mathrm{N}$. & $\mathrm{H}$. & F. & $\mathrm{N}$. & $\mathrm{H}$. & $\bar{F}$. & N. & $\mathrm{H}$. & $\bar{F}$. & $\mathrm{N}$. & $\mathrm{H}$. & $\mathrm{F}$. & $\mathrm{N}$. & $\mathrm{H}$. & $\mathrm{F}$. \\
\hline fumigatus Z5 & 528 & 272 & $99 \%$ & 53 & 98 & $98 \%$ & 26 & 69 & $99 \%$ & 9 & 14 & $100 \%$ & 5 & 61 & $97 \%$ & 12 & 55 & $100 \%$ & 1 \\
\hline fum & 528 & 269 & $100 \%$ & 53 & 109 & $99 \%$ & 27 & 75 & $100 \%$ & 9 & 15 & $100 \%$ & 6 & 62 & $97 \%$ & 11 & 57 & $100 \%$ & \\
\hline A. fumigatus var. RP-2014 & 498 & 257 & $100 \%$ & 53 & 99 & $100 \%$ & 24 & 68 & $100 \%$ & 9 & 15 & $100 \%$ & 6 & 54 & $96 \%$ & 11 & 50 & $100 \%$ & \\
\hline 163 & 532 & 270 & $100 \%$ & 53 & 106 & $99 \%$ & 25 & 73 & $99 \%$ & 9 & 15 & $100 \%$ & 6 & 60 & $97 \%$ & 10 & 58 & $100 \%$ & \\
\hline N. fischeri NRRL 181 & 595 & 304 & $97 \%$ & 54 & 103 & 106 & 25 & 87 & $81 \%$ & 8 & 17 & $88 \%$ & 7 & 78 & $90 \%$ & 12 & 66 & $91 \%$ & \\
\hline
\end{tabular}

Enzymes: GH, glycoside hydrolase; GT, glycosyltransferase; $\mathrm{CE}$, carbohydrate esterase; PL, polysaccharide lyase; CBM, carbohydrate-binding module; AA, auxiliary activities. Genomes: N. fischeri NRRL 181

(GenBank: AAKE00000000.3); A. fumigatus AF293 (GenBank: AAHF00000000.1); A. fumigatus var. RP-2014 (GenBank: JHOI00000000.1); A. fumigatus A1163

(GenBank: ABDB00000000.1). N.: total numbers of each classes; H.: percentage of homologs compared among these five fungi; F.: family numbers of each classes

glycosyltransferases appeared to fulfill a secondary or supportive role in polysaccharides degradation because only a very small percentage of genes were induced, $9.1 \%$.

Plant biomass contains cellulose, hemicellulose, pectin and other natural materials such as lignin, the degradation of which requires many enzymes working synergistically. Cellulose degradation requires endoglucanases (EGs), cellobiohydrolases (CBHs) and $\beta$-glucosidases. EGs catalyze the hydrolysis of cellulose at random positions in less crystalline regions, and $\mathrm{CBHs}$ act processively on the non-reducing ends of the chains to release the disaccharide cellobiose, which is cleaved by $\beta$-glucosidases to yield glucose [12]. A thorough inspection of the Z5 genome revealed at least 25 EGs, 3 CBHs and at least $15 \beta$-glucosidases (Additional file 7). EGs in $\mathrm{Z} 5$ belong to six glycoside hydrolase families (GH5, GH7, GH12, GH16, GH45, AA9), with seven EGs in these families having CBM1 domains (Y699_02044, Y699_02871, Y699_03689, Y699_4295, Y699_05430, Y699_07518 and Y699_08692, respectively). Two CBHs from strain Z5 belong to the family GH7 (CBH1) and another belongs to the family GH6 (CBH2). Z5 has four $\beta$-glucosidases in family GH1 and 11 in GH3. The cellulase genes of A. fumigatus Z5 are not closely adjacent in the genome except for an endoglucanase (Y699_04295) and a cellobiohydrolase (Y699_04296). In addition to the enzymes responsible for the degradation of cellulose, numerous other plant cell wall polysaccharide-degrading enzymes were also predicted in the A. fumigatus Z5 genome, including hemicellulases and pectinases, which have catalytic activities for degradation of hemicelluloses and pectin, respectively (Additional file 7). Analysis of the CAZyme gene locations in the Z5 genome revealed that CAZyme genes were nonrandomly distributed within the genome. Of the 529 CAZyme genes, 209 were located in one of 44 discrete regions, which ranged from $3.6 \mathrm{~kb}$ to $175 \mathrm{~kb}$ in length (roughly $1.8 \mathrm{Mb}$ in total) (Fig. 4 and Additional file 8). Each region contained two to 13 CAZyme genes. Most of the CAZyme genes in the clusters were not from the same subfamily, which indicated that gene relocation rather than duplication was responsible for the formation of the CAZyme gene clusters. Approximately $59.3 \%$ (124) of the CAZyme genes in the clusters encoded glycoside hydrolases. The fact that $46.3 \%$ of the glycoside hydrolase genes and $26.5 \%$ of the glycosyltransferase genes were located in these clusters indicated that the majority of the CAZymes were involved in polysaccharide degradation. Furthermore, of the genes that were differentially expressed in treatment conditions (induction by xylan, cellulose or rice straw) when compared to the sucrose as a control condition, most were located in or around the CAZyme-rich regions (Fig. 5 and Additional file 9).

\section{Protein secretion of $A$. fumigatus $\mathrm{Z5}$}

In contrast to other microorganisms, filamentous fungi can secret large amounts of proteins into the external environment. Strain Z5 is also an extraordinarily efficient producer of extracellular enzymes [25, 26], which makes the analysis of the protein secretion system particular interesting. Here, we compared some components of secretory pathway among strain Z5 and 5 other industrially relevant filamentous fungi extensively used for protein secretion (A. niger, A. nidulans, A. oryzae, $N$. crassa and $T$. reesei). Translocation from the cytoplasm to the endoplasmic reticulum (ER) occurs in these fungi through established signal recognition particle (SRP)dependent and SRP-independent pathways [35], which are very important steps for heterologous gene expression. Orthologs of most $S$. cerevisiae proteins in these two pathways were found in strain Z5 and 5 other fungi except for a yeast signal recognition and docking protein, Srp21p [36]. All these filamentous fungi have a gene encoding protein disulphide isomerase (PDI) (ortho$\log$ of yeast Pdilp) and 3 other PDI-related genes. The chaperone BiP, a member of the heat shock 70 protein family, is more conserved (similarity $>90 \%$ ) than other components of secretory pathways in these fungi, reflecting the central role of $\mathrm{BiP}$ in translocation into the ER, protein folding and regulation response [37, 38]. Multiple enzymes are required for $\mathrm{N}$-glycosylation of glycoproteins, 


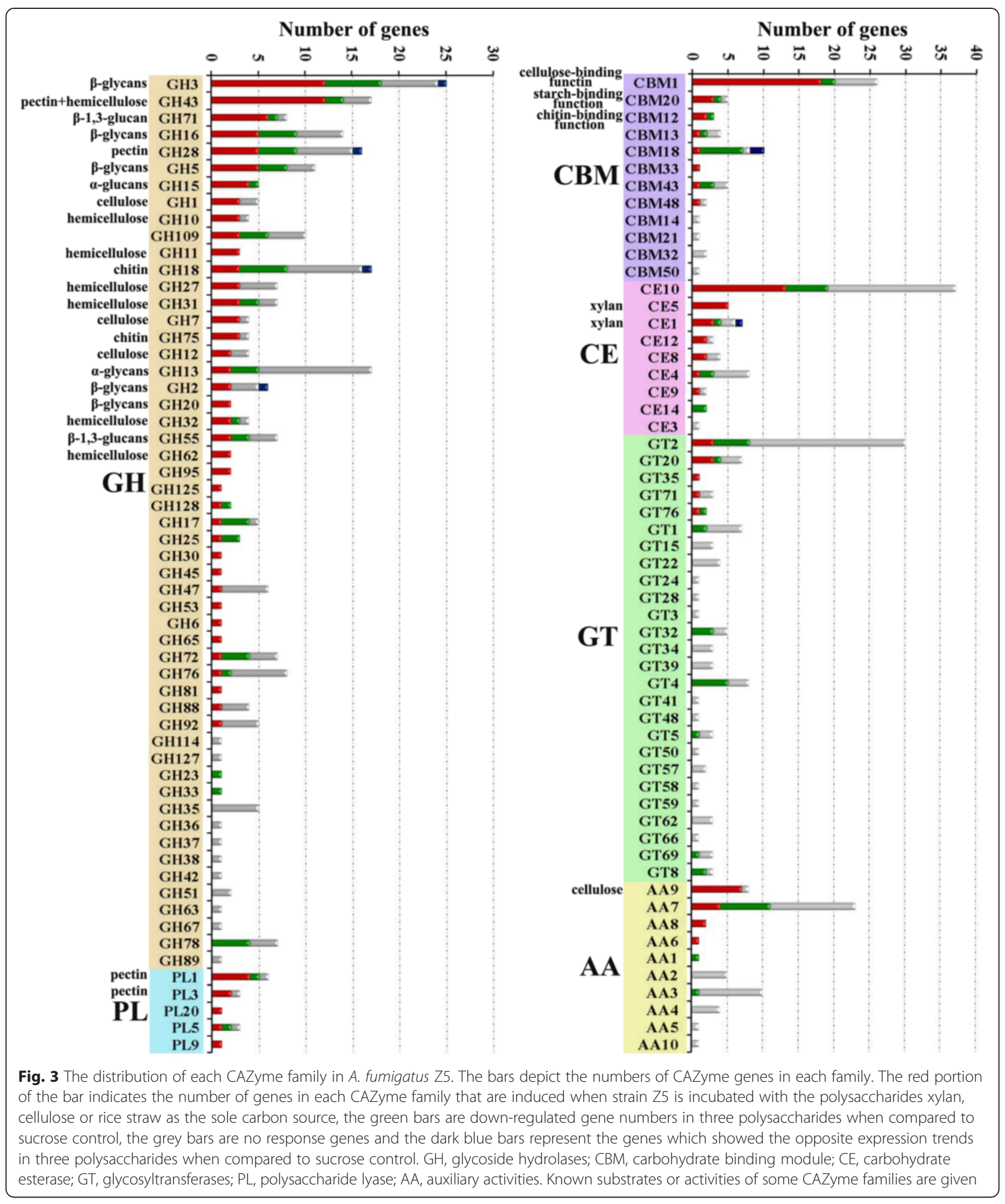

which involves binding of a preformed oligosaccharide (Glc3Man9GlcNAc2) to asparagine side chains of an immature protein. All six fungi have at least one copy of the key enzymes for this process (glucosidase I and II, calnexin, calreticulin and UDP-glucose:glycoprotein glucosyltransferase). which indicates that they may have a common folding and quality control system for glycoproteins. The unfolded protein response (UPR) signaling 

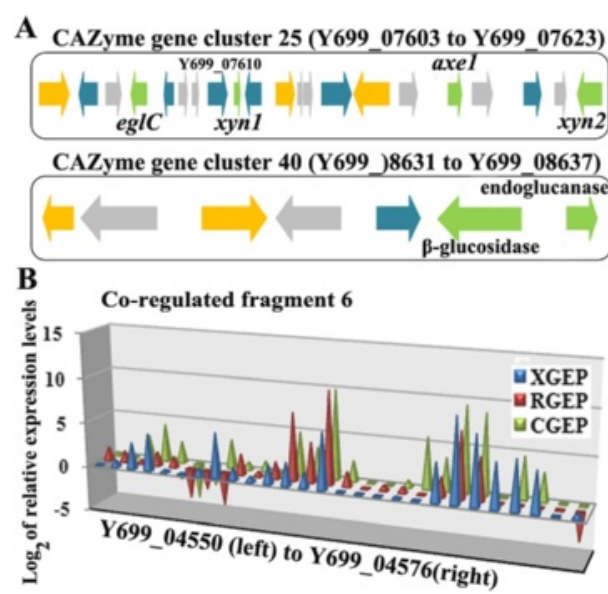

[ CAZyme genes with expressed in XGEP/RGEP/CGEP

naZyme genes with not expressed in XGEP/RGEP/CGEP

Non-CAZyme genes with expressed in XGEP/RGEP/CGEP

Gene model

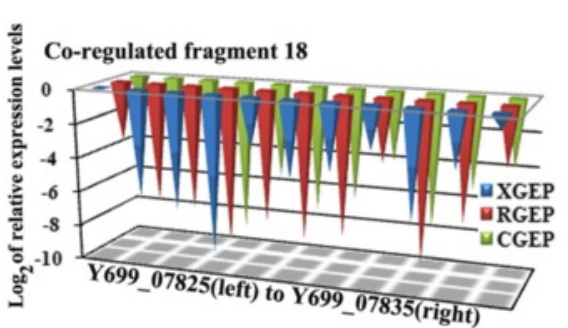

Fig. 4 CAZyme gene clusters and co-regulated fragments. a CAZyme gene clusters: cluster 25 contained a beta-1,3-endoglucanase gene (eglC) and an acetyl xylan esterase gene (axe1), as well as 2 endo-1,4-beta-xylanase genes (xyn1 and xyn2). Y699_7610 encodes an extracellular phytase. In CAZyme gene cluster 40, beta-glucosidase and endoglucanase are co-induced. b Co-regulated fragments: fragment 6 is up-regulated. It includes the CAZyme gene cluster 6 (from Y699_04554 to Y699_04576), in which Y699_04562 encoded a beta-glucosidase, Y699_04563 encoded a MFS sugar transporter, Y699_04570 encoded a xylosidase/arabinosidase and Y699_04574 encoded a MFS alpha-glucoside transporter. Fragment 18 includes the secondary metabolite biosynthetic gene cluster 23 in which genes are down-regulated in all three treatments compared to sucrose. Y699_07834 encoded a polyketide synthase

pathway in these fungi differs somewhat from that of yeast. In mammalian cells, the PERK (double-stranded RNA-activated protein kinase-like endoplasmic reticulum kinase) machinery can attenuate protein translation by phosphorylating the alpha subunit of eukaryotic translation initiation factor 2 (eIF2 $\alpha$ ) in the early phase of ER stress [39]. PERK is then inhibited by binding to the HSP40 cochaperone p58, the expression of which is induced by IRE1/XBP1 of the UPR pathway. The ortholog of p58 was found in the genomes of these fungi but not in yeast, and the ortholog of PERK was not found or had low similarity. Homologs of most known ERAD components were found in all these filamentous fungi, despite an apparent lack of orthologs and little sequence similarity to yeast ERAD components, as reported for the A. niger genome [36]. Some counterparts of the mammalian GTPase proteins (Rab2, Rab4, Arf6 and Arf10), which are involved in membrane fusion and vesicle budding in diverse cellular locations, were found in all these fungi, whereas they were absent in yeast [40].

In order to get insight into the secretory pathway of $A$. fumigatus $\mathrm{Z} 5$, transcriptional profiles of genes in the secretory pathway induced by different carbon sources were analyzed (Additional file 10). Most genes had higher expression levels when grown on sucrose than on the other three carbon sources (xylan, cellulose and rice straw as the sole carbon source). This is interesting because the samples with more extracellular proteins have low gene transcription in protein synthesis. qRTPCR results of several selected genes verified the transcriptomic data (Fig. 6a). These results indicated that the polysaccharides-induced samples actually had a relative low stress of protein synthesis when compared to sucrose control. Sucrose, as a favored carbon source, could promote the growth of strain Z5 much faster than other polysaccharides.

\section{Transcriptional responses of $A$. fumigatus $\mathbf{Z 5}$ to different carbon sources}

Genome analysis showed the potential of a wide spectrum of polysaccharide hydrolytic enzymes produced by $A$. fumigatus Z5. In order to determine which hydrolytic enzyme-encoding genes were induced by different substrates (sucrose, xylan, cellulose and rice straw), the transcriptional profiles of $A$. fumigatus $\mathrm{Z} 5$ under these conditions were determined. Xylan, as the major component of hemicellulose, was used as a sole carbon source to induce the expression of hemicellulase genes, while cellulose was used as the inducer of cellulases. Rice straw, which contains various polysaccharide components, was chosen as a carbon source to study the diversity of the degradation enzymes. A. fumigatus Z5 was grown on $2 \%$ sucrose (used as control), and the mycelia were transferred to $1 \%(\mathrm{w} / \mathrm{v})$ xylan, cellulose or rice straw as the sole carbon source for $16 \mathrm{~h}$. Gene expression patterns revealed that comparable genes were differentially expressed in each transcriptome, and many had similar patterns (Figs. 7 and 8). The xylan-induced gene expression pattern (XGEP) is relatively similar to the sucroseinduced gene expression pattern (SGEP), which may be due to the high efficiency of xylan degradation (data not shown) and xylose-induced carbon catabolite repression [41, 42]. However, the cellulose-induced gene expression pattern (CGEP) and the rice straw-induced gene 


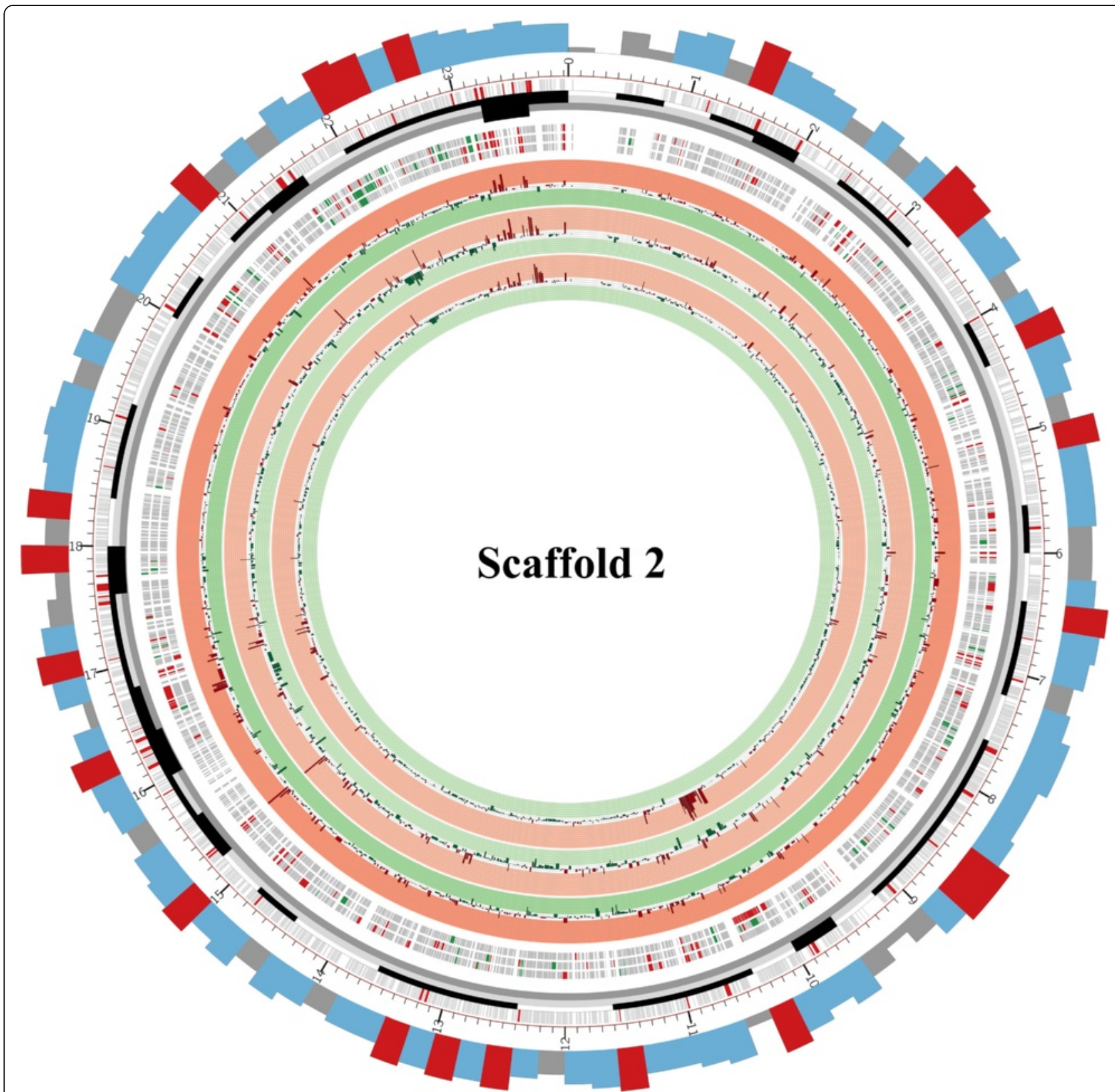

Fig. 5 Map of scaffold 2. The first (outermost) circle represents the gene density of scaffold 2. The red indicates gene number higher than 8 in that $20 \mathrm{~kb}$ fragment, the blue indicates gene number from 6 to 8 in that $20 \mathrm{~kb}$ fragment, and the grey indicates gene number below 6 in that $20 \mathrm{~kb}$ fragment. The second circle represents scaffold size, and the smallest unit represents $10 \mathrm{~kb}$. The third circle represents all genes in scaffold 2. CAZyme genes are indicated in red. The discrete black boxes describe the density of CAZyme genes. The next three circles indicate the genes which expression differs significantly in xylan, rice straw or cellulose, from outside to inside, respectively, when compared to sucrose as a control condition. Up-regulated genes are red, and down-regulated genes are green. The innermost three circles are gene expression graphs in cellulose, rice straw and xylan relatively to sucrose, from outside to inside, respectively. A red background indicates $\log _{2}$ (ratio of RPM values) $\geq 2$, and a green background indicates $\log _{2}$ (ratio of RPM values) $\leq-2$ ). Scaffold 2 has a CAZyme gene cluster that spans the bases $2300-2370 \mathrm{~kb}$ in which all genes were up-regulated relatively to sucrose in the 3 treatments

expression pattern (RGEP) differ significantly from the SGEP (Fig. 7b, c). The main aim of this study was to focus on genes that have significantly different expression among the 4 different treatments. Four hundred and two genes were identified in cluster 3 and cluster 4 , which showed higher expression levels in the XGEP, CGEP and RGEP than in the SGEP. These two clusters contained nearly all of the polysaccharides-degrading genes, including 14 cellulases (7 EGs: Y699_00150, Y699_02424, Y699_05430, Y699_06174, Y699_07518, Y699_08637 and 


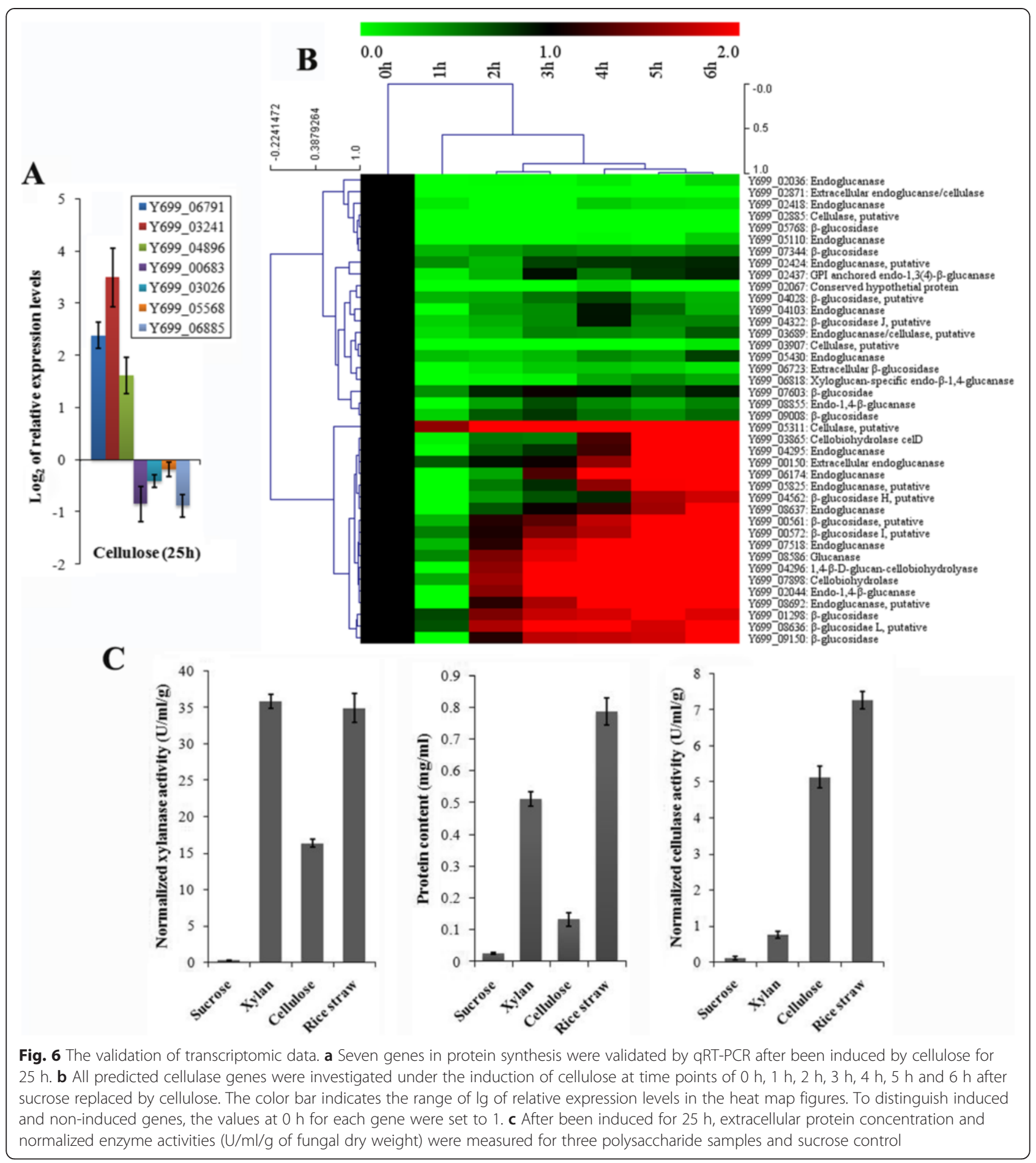

Y699_08692; 2 CBHs: Y699_03865 and Y699_07898; 3 glucosidases: Y699_00561, Y699_01298 and Y699_08636), nine xylanases (5 endo-1,4-beta-xylanases: Y699_04481, Y699_06333, Y699_07611, Y699_07623 and Y699_09486; 4 ß-xylosidases: Y699_04570, Y699_04662, Y699_05610 and Y699_07880), 2 alpha-L-arabinofuranosidases and 2 acetyl xylan esterases. Seven other cellulases (3 EGs:
Y699_02044, Y699_04295 and Y699_05825; $4 \quad \beta$ glucosidases: Y699_00572, Y699_04562, Y699_06723 and Y699_09150) were found in cluster 6 and were expressed at higher levels in the CGEP and RGEP than in the SGEP and XGEP. Together, these results show that both cellulose and xylan can induce the expression of cellulase and xylanase genes, but xylan induces fewer cellulase genes 


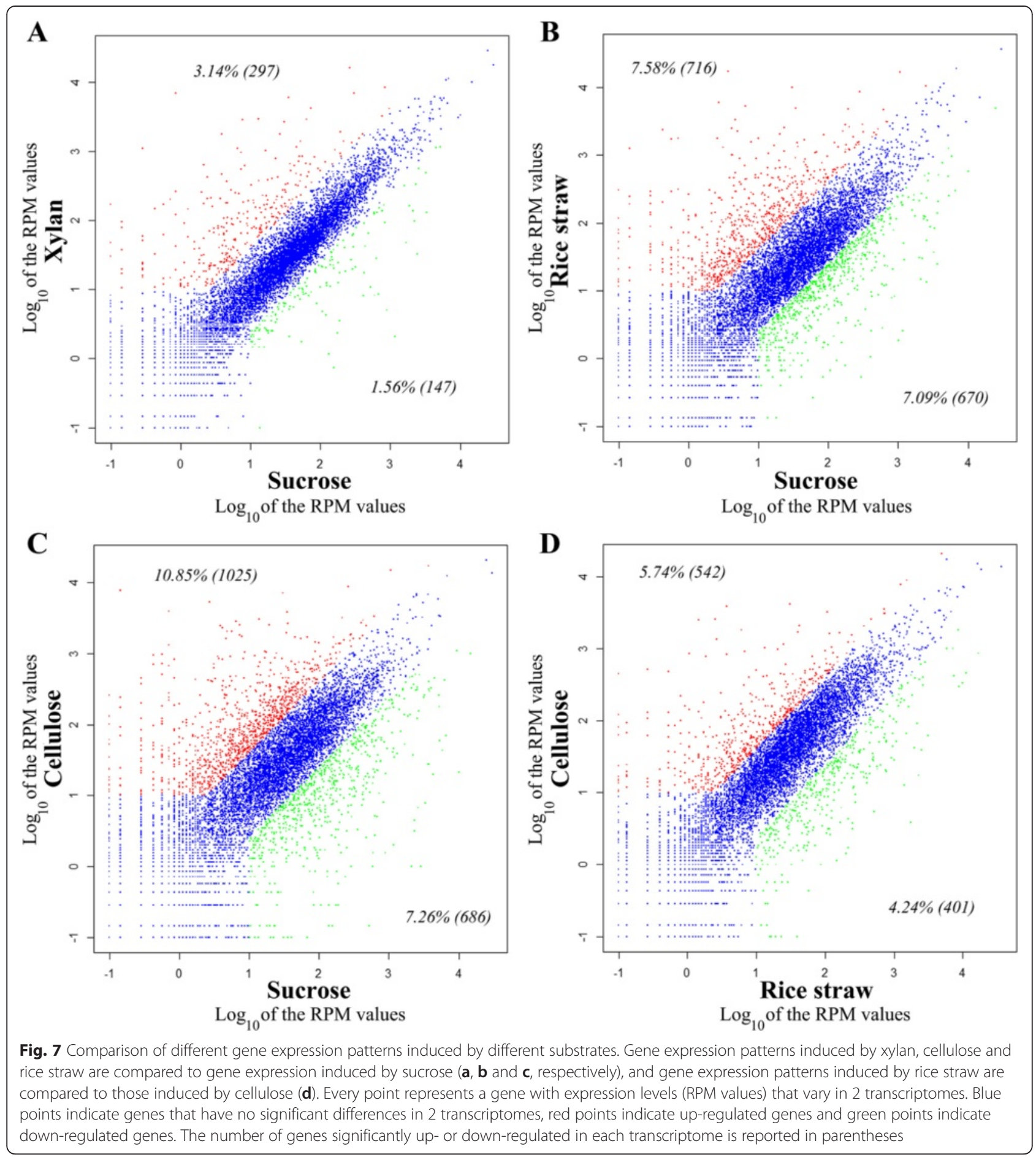

(Fig. 9). Three cellobiose dehydrogenase (cdh) genes (Y699_02120, Y699_04685 and Y699_05507) were detected in the A. fumigatus Z5 genome. Consistent with a recent report that cellobiose dehydrogenase and a copperdependent polysaccharide monooxygenase can enhance cellulose degradation [43], we found that the $c d h$ gene (Y699_02120) was induced by the substrates cellulose and rice straw. Gene Y699_04685 in cluster 2 only had a high expression level in RGEP compared to the three other patterns, suggested that this cellobiose dehydrogenase may not functional in the degradation of cellulose and xylan but may be useful for degradation of other components of plant biomass, such as pectin or other aromatic substrates. Interestingly, two endoglucanase genes (Y699_ 02418 and Y699_05110) were also located in cluster 2, which indicated that these two genes may not functional 


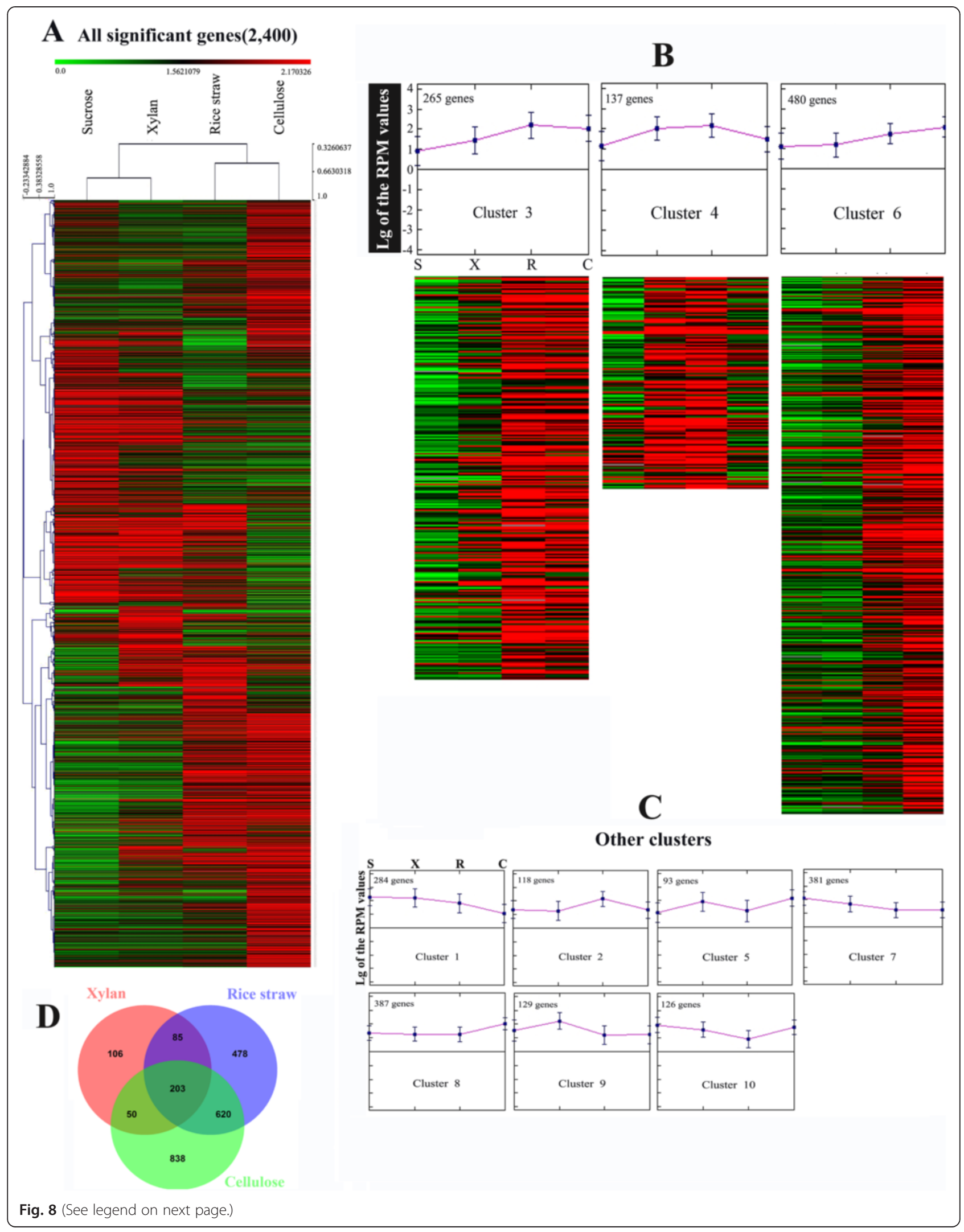


(See figure on previous page.)

Fig. 8 Gene expression profiles for substrate-responsive genes. The color bar indicates the range of expression levels in the heat map figures a Genes with significantly different expression are depicted (see Methods). b Three clusters are depicted with heat map figures and centroid graphs. In cluster 3 and cluster 4, genes are expressed at a higher level in all 3 treatments (xylan, cellulose and rice straw) compared to sucrose. In cluster 6 genes are only highly expressed when growing in cellulose and rice straw. c The other seven clusters, Vertical bars on graphs represent the data range, and the points in the middle represent the average. $\mathbf{d}$ Venn diagram obtained from the comparison of the significantly differently expressed genes induced by different polysaccharides when compared to sucrose control

in the degradation of cellulose due to their low expressional levels in the CGEP.

To validate the transcriptomic results, all the predicted cellulase genes were investigated by qRT-PCR under the induction of cellulose, which confirmed the transcriptomic results (Fig. 6b). Additionally, extracellular protein contents and total xylanase and cellulase activities of the 4 treatments (sucrose, cellulose, xylan and rice straw) provide further evidences to the transcriptomic data (Fig. 6c).

The MFS includes 82 protein families, 6 of which are known to transport sugars (the sugar porter (SP) family, the oligosaccharide: $\mathrm{H}^{+}$symporter (OHS) family, the fucose: $\mathrm{H}^{+}$symporter (FHS) family, the sialate: $\mathrm{H}^{+}$ symporter (SHS) family, the polyol permease (PP) family and the sugar efflux transporter (SET) family), in addition, the glycoside-pentoside-hexuronide (GPH):cation symporter family is also responsible for sugars transportation [44]. Totally, strain Z5 has 88 sugar transporter genes distributed among SP, FHS, SHS and GPH families (SP family includes 79 genes), 37 genes were differently expressed in polysaccharides-induced samples when compared to sucrose control, the 8 genes which expression changed the most were predicted to transport glucose, xylose, lactose and maltose. Three (Y699_03783, Y699_ 04557 and Y699_04571) and six sugar transporter genes
(Y699_01300, Y699_02331, Y699_03701, Y699_07512, Y699_09366 and Y699_09270) were only induced by xylan and cellulose, respectively. Gene Y699_09366 was up-regulated about 200 folds by both cellulose and rice straw, which is the homolog of a lactose permease gene (Gene ID: 3450) essential for cellulase induction in T. reesei v2.0 [45]. All the homologs of the 37 genes could be detected in other 5 filamentous fungi $(A$. niger, $A$. nidulans, A. oryzae, $N$. crassa and T. reesei) even though $N$. crassa only has 44 sugar transporter genes, which indicated that filamentous fungi shared common mechanisms of sugar transportation.

Strain Z5 has 7246 and 5569 homologous genes with $A$. niger and $N$. crassa respectively. Comparison of the transcriptomic data of Z5, A. niger (GEO DataSets series: GSE57315) and N. crassa (GEO DataSets series: GSE42692) under the induction of different polysaccharides (glucose served as control for A. niger, sucrose for stain $\mathrm{Z} 5$ and $N$. crassa) found that avicel- and rice straw-induced transcriptomic profiles of strain Z5 were very similar, and orange peel power (OPP)- and pectininduced transcriptomic profiles of $N$. crassa were similar (Fig. 10a), however, the responses of the three fungi to the same polysaccharide were significantly different, xylaninduced transcriptomic profile of strain Z5 was similar to avicel-induced gene transcriptomic profile of $N$. crassa.


Fig. $\mathbf{9}$ The conditions of degrading-enzyme gene expression. The whole histogram includes all genes in that group, and black indicates the genes that were significantly different in that treatment compared to sucrose. a CAZyme gene numbers with significantly different expression levels in xylan, rice straw and cellulose when compared to sucrose control. b Hydrolase genes with significantly different expression levels in xylan, rice straw and cellulose compared to sucrose 

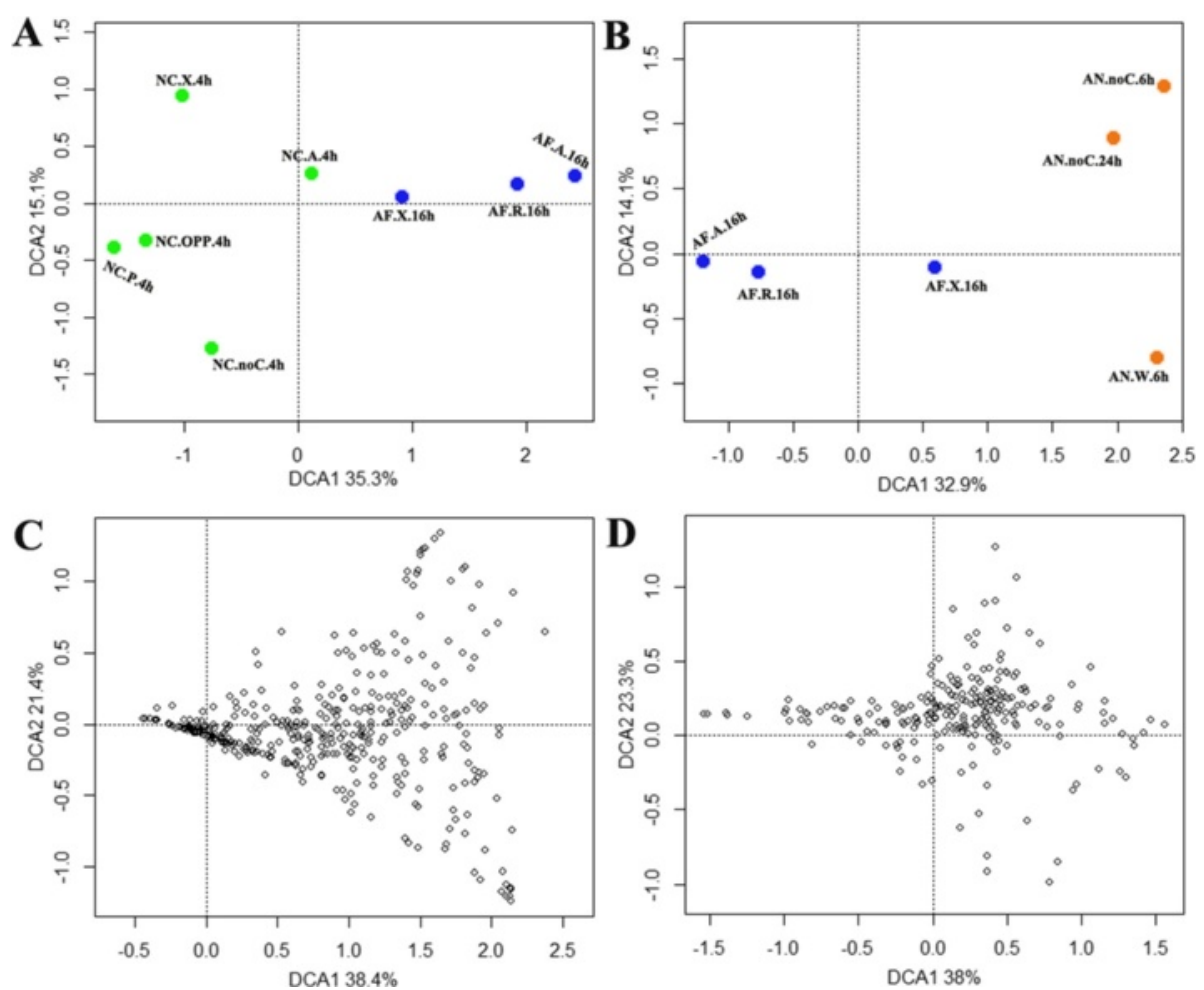

Fig. 10 Comparison of different fungal expression profiles induced by polysaccharides. Expression profiles induced by polysaccharides were compared between strain Z5 and other two filamentous fungi, N. crassa and A. niger, by the method of detrended correspondence analysis. The percentages of the first two principal components explaining the expressional variations were given in axis tags. a Expression profiles of 5557 homologous genes were compared between strain Z5 and N. crassa. AF.X.16h, AF.A.16h and AF.R.16h: induction by xylan, avicel and rice straw respectively for $16 \mathrm{~h}$ in A. fumigatus Z5. NC.X.4h, NC.A.4h, NC.P.4h, NC.OPP.4h and NC.noC.4h: induction by xylan, avicel, pectin, orange peel power and no carbon respectively for $4 \mathrm{~h}$ in $N$. crassa. $\mathbf{b}$ Expression profiles of 7246 homologous genes were compared between strain $Z 5$ and A. niger. AN.W.6h and AN.noC.6h: induction by wheat straw and no carbon respectively for $6 \mathrm{~h}$ in A. niger. AN.noC.24h: induction by no carbon for $24 \mathrm{~h}$ in A. niger. c 392 homologous CAZyme genes were compared between strain Z5 and A. niger, which were induced by polysaccharides and no carbon (shown up). d 241 homologous CAZyme genes were compared between strain Z5 and N. crassa

The 7246 homologs of strain Z5 and A. niger include 391 CAZymes, some of which had the similar responses to the same polysaccharide (Fig. 10c), this trend was also observed between $N$. crassa and strain Z5 (Fig. 10d). Comparison of the straw induced transcriptomic profiles of different strains, transcription of 1386 genes were significantly changed by the induction of rice straw in strain Z5 when compared to sucrose, however, in A. niger and T. reesei, only 514 and 529 genes' transcription were changed (defined by the same standard), respectively, by the induction of wheat straw [22, 46]. Among these differently expressed genes, only 154/94 homologs were found between strain Z5 and A. niger/T. reesei (Additional file 11), and most of these homologs were cellulases, xylanases, sugar transporters and hypothetical proteins. In general, filamentous fungi showed the diverse performances to plant biomass in the whole transcriptome level, but they shared a fair amount of similarity on plant biomass degradation.

\section{GO enrichment analysis}

Analysis of expression patterns in different treatments indicated that 444, 1386 and 1711 genes were up-regulated or down-regulated in the XGEP, RGEP and CGEP, respectively, in comparison to the SGEP (Fig. 8d). A GO term enrichment analysis of the three polysaccharide-induced gene expression patterns compared to the SGEP was performed with the topGO $\mathrm{R}$ package (Additional file 12) [47]. XGEP vs. SGEP analysis showed enrichment of transmembrane transport $(\mathrm{P}=1.1 \mathrm{e}-05)$, the $\mathrm{D}$-xylose metabolic process $(\mathrm{P}=1.3 \mathrm{e}-04)$, the xylan catabolic process $(\mathrm{P}=7.5 \mathrm{e}-04)$ and the polysaccharide binding category $(\mathrm{P}=9.3 \mathrm{e}-04)$. Comparison of RGEP with SGEP indicated enrichment of transmembrane transport $(\mathrm{P}=$ 1.4e-06), the cellulose catabolic process $(\mathrm{P}=6.0 \mathrm{e}-05)$, the xylan catabolic process $(\mathrm{P}=8.4 \mathrm{e}-06)$, the pectin catabolic process $(\mathrm{P}=5.5 \mathrm{e}-05)$ and the polysaccharide binding category $(\mathrm{P}=5.8 \mathrm{e}-12)$. The comparison of CGEP with SGEP revealed enrichment of carbohydrate transport 
( $\mathrm{P}=7.5 \mathrm{e}-04)$, the cellulose catabolic process $(\mathrm{P}=7.6 \mathrm{e}-05)$, the hemicellulose metabolic process $(\mathrm{P}=7.5 \mathrm{e}-04)$, the polysaccharide binding category $(\mathrm{P}=6.2 \mathrm{e}-10)$ and ribosome biogenesis $(\mathrm{P}=1.4 \mathrm{e}-18)$. Together, these results show that $A$. fumigatus Z5 could adjust its gene expression and metabolic pathways by using different substrates as a carbon source. When xylan was supplied, Z5 had a higher expression level of xylanase genes and carbohydrate transporter genes, which could degrade xylan into xylobiose or xylose and then transport them into the cell. Xylobiose and/or xylose could be utilized to produce energy or synthesize other materials in the D-xylose metabolic process. Degradation enzymes, transporters and polysaccharide binding activity were also needed during the degradation of cellulose and rice straw. Intriguingly, CGEP showed enrichment of ribosome biogenesis, indicating that A. fumigatus Z5 needs more ribosomes for protein synthesis. The protein products might be secreted into the external environment and contribute to the difficult process of crystalline cellulose degradation.

Analysis of the transcriptome profiles across all scaffolds (Fig. 5 and Additional file 9) indicated that the expression levels of most genes were stable, while significant changes occurred in and around CAZyme-rich regions. A total of 468 genes were found in the 35 fragments; they ranged from $8 \mathrm{~kb}$ to $112 \mathrm{~kb}$ in length, and all were up-regulated or down-regulated in at least 1 of the 3 transcriptomes (Fig. 4 and Additional file 13). Among these fragments, eight segments corresponding to eight different CAZyme gene clusters were found, and five of them were up-regulated in all three transcriptomes compared to SGEP, while the others were downregulated. These results suggested that the genes in and around the eight CAZyme gene clusters might be closely related to utilization of different carbon sources. Additionally, 11 of the 30 secondary metabolite biosynthetic gene clusters (Additional file 14) in the genome were located or partly located in the co-regulated fragments. Of those 11, five were up-regulated, and the remainders were down-regulated. Therefore, the secondary metabolism pathways might be stimulated in A. fumigatus Z5 when the complex organic substances exist in the environment, enabling the strain Z5 to fend off competition for nutrients and survive in complex environmental conditions.

\section{Discussion}

Filamentous fungi efficiently degrade plant biomass and secret substantial amounts of extracellular enzymes into their substrates. Several model filamentous fungi, including N. crassa, T. reesei, A. niger, A. oryzae and A. nidulans, are widely used in industry and have been the subjects of numerous studies [16, 31, 36, 40, 48]. Unlike the three model species in the Aspergillus genus that have a long history of safe use for industrial enzyme production, A. fumigatus strains are opportunistic pathogens that may cause invasive aspergillosis in immunocompromised patients [29, 49], although strains in this species are also effective at biomass degradation. A. fumigatus $\mathrm{Z} 5$ was isolated from crop compost heaps [26], suggesting that the strain is widely distributed in the environment and contributes to the degradation of plant biomass. There have been some studies related to polysaccharide degradation in Aspergillus fumigatus $[24,26,50,51]$. This study on strain Z5 showed that it has an abundant distribution of CAZyme genes in genome and can produce large amount of extracellular enzymes, which make it possible to be a producer of enzymes to degrade plant biomass. Also, as a fast growing filamentous fungus at a high temperature of $50{ }^{\circ} \mathrm{C}$, strain Z5 could easily be a host for heterologous protein expression by the mature molecular technologies in $A$. fumigatus. Even though, strain Z5 was not as good as some well-studied fungi, such as A. niger MGG029 with many genomic modifications [49], but it shared a similar potential and results of the present study enable valuable resources for further explorations. The safety of this strain should be considered by knocking out some virulence-related genes, such as the genes for gliotoxin, fumagillin, aflatoxin and ribotoxin [52].

In general, filamentous fungi cannot absorb polysaccharides directly into their cells to use as sources of carbon and energy. Instead, they secrete many types of enzymes to depolymerize polysaccharides into monomeric carbohydrates that are efficiently taken up and metabolized. In eukaryotic cells, protein secretion involves ER-associated translation, folding and glycosylation. Proteins are then moved into the Golgi apparatus or other compartments via vesicles to form the mature proteins. Finally, within secretory vesicles, the mature proteins are transported to the cell membrane and secreted into the environment [53]. Guillemette et al. [54] reported transcriptional responses of $A$. niger when exposed to secretion stress (treatment with tunicamycin, treatment with DTT or forced secretion of a heterologous protein). In total, 11 genes in the secretory pathway were induced by the three ER-stress conditions. However, among the homologous counterparts of these 11 genes in A. fumigatus Z5, seven were down-regulated in all three treatments (xylan, cellulose and rice straw) when compared to the sucrose as a control condition (Additional file 10). The same trend was observed among the important chaperones and foldases (bipA, pdiA and $c l x A$ ), which are involved in protein folding in the ER, with the exception of a 1.3-fold increase in bipA observed on cellulose. Unfolded protein response (UPR) and ERassociated degradation (ERAD) were also not induced by 
the three polysaccharides. These results indicate that there was no ER stress in these conditions compared to sucrose, even though more extracellular proteins were secreted [53]. Two genes involved in glycosylation, the $\alpha-1,2$-mannosidase gene (Y699_04911) and the $\alpha$-glucosidase gene (Y699_09020), exhibited 1.522.3-fold and 3-76.4-fold increases, respectively, in the three treatments compared to sucrose control. It has been suggested that plant biomass-degrading enzymes are highly glycosylated [55]. Our study indicates that when polysaccharides are the sole substrate for growth, high levels of extracellular enzymes do not cause ER stress. Indeed, it suggested that if the balance between growth and protein secretion of fungi is managed well, the secretory pathway can be fully explored, and foldincreased production of extracellular proteins can be achieved.

$\mathrm{X} \ln \mathrm{R}$ is a xylanolytic transcriptional activator specific to fungi [56] that effects a wide range of target genes, e.g., genes encoding xylan-degrading enzymes or the enzymes in the D-xylose metabolic pathway $[57,58]$ and even genes encoding cellobiohydrolases and endocellulases [59]. In this study, the $\mathrm{X} \ln R$ gene was significantly up-regulated by cellulose and rice straw but not by xylan. Andersen et al. [60] reported that in the fermentations of glucose and xylose, 22 conserved genes were differentially transcribed (21 up-regulated and one downregulated) in three Aspergillus species (A. niger, A. oryzae and $A$. nidulans) when xylose, rather than glucose, was the substrate. Enzymes involved in D-xylose metabolism were among the 22 genes. In the present study, homologs of the 21 up-regulated genes were mostly transcribed at high levels in the three treatments compared with sucrose control (Additional file 15). However, the results indicated that $\mathrm{D}$-xylose metabolism was not activated by $\mathrm{X} \ln \mathrm{R}$ because its transcriptional level was similar in xylan and sucrose, even though the D-xylose metabolic pathway was significantly induced in xylan. The down-regulated gene identified by Andersen et al. encoded a MFS monosaccharide transporter, which was reported to have a higher affinity for glucose than xylose in their results. The expression level of the homolog gene decreased considerably in the three polysaccharides treatments of this study, suggesting that either the cellulose and rice straw treatments did not have enough glucose to induce the transporter gene or cellulose was transported into cells mainly as a form of cellobiose rather than glucose [61]. Another interesting finding in the 22 genes is that a sugar transporter (Y699_05888) had a extremely high expression level in xylan and rice straw (81.9-folds and 64.6 -folds, respectively) but only a 4.7 -folds enhanced in cellulose, which revealed that this sugar transporter should be a specific transporter of the hydrolyzing products from xylan in all four Aspergillus species (qRT-PCR validated that gene Y699_05888 was up-regulated $2.8 \pm 0.08$ and $27.2 \pm 0.52$ folds by cellulose and xylan, respectively).

Transcriptomic data showed that genes encoding hydrolytic enzymes involved in plant cell wall degradation were repressed during growth on sucrose. Carbon catabolite repression (CCR) is an important mechanism to repress the production of polysaccharide-degrading enzymes during growth on easily metabolized carbon sources. In most fungal species, CCR is mediated by a zinc-finger transcription factor CreA [62-64], which is a repressor for genes encoding polysaccharide-degrading enzymes. In this study, we expected to have low CreA expression levels in the 3 polysaccharides treatments when compared to sucrose treatment. However, the creA gene was transcribed at high levels $(\mathrm{RPM}=4714.2$ in rice straw and RPM $=3366.3$ in cellulose), with 7.7- and 5.5fold increases in rice straw and cellulose compared to sucrose, respectively. Reverse transcription quantitative PCR (RT-qPCR) experiments under the same conditions as the transcriptomic study confirmed these observations (data not shown). These results suggest that CreA has functions beyond those of a transcription repressor and merits further research.

Our previous research identified many plant biomassdegrading enzymes secreted by Z5 in the presence of different carbon sources (i.e., glucose, cellulose and rice straw) [25]. The expression of those enzymes in the present study is consistent with the findings of the previous study. Additionally, xylanase genes were induced more by cellulose and rice straw than by xylan; xylan was not the best inducer of its own hydrolytic enzymes. We observed that cultivation broth containing xylan became transparent, while culture filtrates with avicel or rice straw still had unused substrates after $16 \mathrm{~h}$ of incubation, indicating that pure xylan was depolymerized faster than avicel and rice straw; our previous work showed that Z5 has very strong xylanase activities [25]. We conclude that the simple carbon source, xylose, which is released by xylan degradation, inhibited the persistent high expression of many xylanases. This may also account for the smaller transcriptional differences between the xylan treatment and sucrose control than those observed when either cellulose or rice straw treatments are compared to the control.

\section{Conclusions}

This study performed transcriptional analysis on an $A$. fumigatus strain grown on xylan, cellulose and rice straw. It comprehensively described natural polysaccharide saccharification by $A$. fumigatus hydrolases and the regulation of gene expression that occurs during this process. Having identified enzymes actively involved in 
biomass degradation, we foresee the potential to increase enzyme activities in industrial contexts. This would first require the heterologous expression of the enzymes at high levels and optimization of their incorporation into enzymatic cocktails. The genome and transcriptome data of this study are also valuable for future studies of aspergillosis.

\section{Methods}

\section{Genome assembly, scaffolding, and gene prediction}

The genome of A. fumigatus strain Z5 was shotgun sequenced using a Roche 454 GS FLX system at the Chinese National Human Genome Center (Zhangjiang Hi-tech Park, Shanghai, China). It produced $837 \mathrm{Mb}$ of sequence data $(29.9 \times$ coverage $)$ with an average read length of $358 \mathrm{bp}$. Using the Newbler software (v2.3) within the Roche 454 suite package [65], these reads were assembled into 596 contigs with a total size of 29.4 Mb. For sequence scaffolding, Solexa Mate Pair reads (insert size $3 \sim 5 \mathrm{~kb}$ ) were used to establish the genome scaffolds. A total of $38,248,825$ mate-pair reads contributed to improving sequence quality and constructing scaffolds. A simple greedy algorithm was used to optimize the order of the contigs and provided a feasible heuristic solution for scaffolds construction [66]. By mapping the reads to contigs, 557 contigs were then linked, generating 24 genome scaffolds with their length not smaller than $1 \mathrm{~kb}$. For gene prediction, Genemark (v2.3 a, http://topaz.gatech.edu/GeneMark/) and Augustus (v2.5, http://bioinf.uni-greifswald.de/augustus/) were used to predict genes for the Z5 genome, and the genome of $A$. fumigatus AF293 was used as a reference. The predicted genes were annotated using public databases (NCBI nr, Uniprot and KEGG) with BLAST, and the predicted genes with the best supporting annotation results were selected as the gene models. As a result, a total of 9540 gene models were described in the genome. Transfer RNAs (tRNAs) were predicted with tRNAscan-SE [67] (http:// lowelab.ucsc.edu/tRNAscan-SE/). Secreted proteins were identified by SignaIP 4.0 analysis [68] (http://www.cbs. dtu.dk/services/SignalP/).

\section{Strains and culture conditions}

Strain Z5 was grown on potato dextrose agar (PDA) plates for conidia production. Conidia were harvested by washing the plate with $10 \mathrm{ml}$ sterile $\mathrm{ddH}_{2} \mathrm{O}$. After removing the mycelia, the conidia were resuspended, and the concentration was adjusted to $1 \times 10^{7}$ conidia/ $\mathrm{ml}$. Basic culture medium (BCM) was composed of the following: $0.25 \mathrm{~g} / \mathrm{L}$ yeast extract, $1 \mathrm{~g} / \mathrm{L} \mathrm{MnSO}_{4} \cdot \mathrm{H}_{2} \mathrm{O}, 0.5$ $\mathrm{g} / \mathrm{L}$ urea, $0.5 \mathrm{~g} / \mathrm{L}\left(\mathrm{NH}_{4}\right)_{2} \mathrm{SO}_{4}, 0.5 \mathrm{~g} / \mathrm{L} \mathrm{MgSO}_{4} \cdot 7 \mathrm{H}_{2} \mathrm{O}, 7.5$ $\mathrm{mg} / \mathrm{L} \mathrm{FeSO} \mathrm{O}_{4} \cdot 7 \mathrm{H}_{2} \mathrm{O}, 2.5 \mathrm{mg} / \mathrm{L} \mathrm{MnSO}_{4} \cdot \mathrm{H}_{2} \mathrm{O}, 3.6 \mathrm{mg} / \mathrm{L}$ $\mathrm{ZnSO}_{4} \cdot 7 \mathrm{H}_{2} \mathrm{O}, 3.7 \mathrm{mg} / \mathrm{L} \mathrm{CoCl}_{2} \cdot 6 \mathrm{H}_{2} \mathrm{O}, 0.5 \mathrm{~g} / \mathrm{L} \mathrm{CaCl}_{2}$ and a predetermined concentration of carbon source according to our experimental conditions [69]. $1 \times 10^{7}$ conidia/ $\mathrm{L}$ were inoculated into liquid $\mathrm{BCM}$ with sucrose as the carbon source (BCM-S) and cultured at $45^{\circ} \mathrm{C}$ for $24 \mathrm{~h}$. For cultivation in a medium containing xylan from oat spelts (Sigma), Avicel PH-101 (Sigma-Aldrich) or rice straw as a carbon source, the mycelia grown in the BCM-S were exhaustively washed with sterile distilled water and then transferred into yeast-free BCM and containing $1 \%$ oat spelts xylan, $1 \%$ Avicel PH-101 (a source of cellulose), or $1 \%$ rice straw as a carbon source for 16 h. Mycelia were harvested by filtration through one layer of gauze, washed thoroughly with sterile water and quickly frozen in liquid nitrogen for further RNA extraction. The reason for sucrose used to be control is that sucrose is a favored carbon source to polysaccharides, and it will not induce any lignocellulolytic genes in filamentous fungi. For BCM with rice straw, the rice straw obtained from the farmland was exhaustively washed with deionized water for several days until reducing sugars were not detected by dinitrosalicylic acid (DNS). The rice straw was then dried completely at $40{ }^{\circ} \mathrm{C}$, made into rice straw powder using a grinder and stored at room temperature.

\section{Enzyme assays}

Extracellular proteins were precipitated by $80 \%$ ammonium sulfate and redissolved in sterile distilled water. The protein concentration was determined with a Micro $\mathrm{BCA}$ protein assay kit (Beyotime, China) using bovine serum albumin as a standard. Cellulase and xylanase activities were measured by 3,5-dinitrosalicylic acid (DNS) method according to Liu et al. [25]. One unit of enzyme activity was defined as the amount of enzyme required to release $1 \mu \mathrm{mol}$ of reducing sugars from the substrate in $1 \mathrm{~min}$.

\section{RNA extraction and qRT-PCR experiments}

The frozen mycelia were disrupted by grinding in liquid nitrogen, and total RNA was extracted using the RNeasy Plant Mini Kit (Qiagen) combined with the RNase-Free DNase set (Qiagen). The total RNA (approximately 2 $\mu \mathrm{g})$ extracted from each treatment was fractionated on a $1.2 \%$ agarose gel, stained with ethidium bromide and visualized with UV light to check RNA integrity. The total RNA samples were then quantified using a NanoDrop 2000 spectrophotometer (Thermo, USA). For qRTPCR analysis, cDNA were synthesized from the total RNA in different induction time using a PrimeScript ${ }^{\text {in }}$ RT-PCR Kit (TAKARA, DaLian, China) according to the manufacturer's instructions. All quantitative PCR runs were performed in triplicate on a 7500 Fast Real-Time PCR System (Applied Biosystems) using SYBR ${ }^{\bullet}$ Premix Ex Taq $^{\text {Ti }}$ Kit (Tli RNaseH Plus) (TAKARA, DaLian, China). An actin-encoding gene and a histone-encoding gene 
(GenBank Accession No: Y699_04988 and Y699_07225, respectively) were chosen together to be reference genes. The primer sequences are given in Additional file 16.

\section{Transcriptome sequencing and gene mapping}

Messenger RNA was purified from the total RNA with a Micropoly(A)PuristTM mRNA purification kit (Ambion, America). Double-stranded cDNA was synthesized according to Ng's full-length cDNA synthesis protocol [70] with some modifications. GsuI-oligo dT was used as the primer for first-strand cDNA synthesis from $10 \mu \mathrm{g}$ of mRNA, using 1,000 units of Superscript II reverse transcriptase (Invitrogen, America). The diol group of the CAP structure of mRNA was then oxidized by $\mathrm{NaIO}_{4}$ (Sigma, America) and linked to biotin hydrazide. Dynal M280 streptavidin Dynabeads (Invitrogen, America) were used to select biotinylated RNA/cDNA. The first-strand cDNA was then released by alkaline hydrolysis. Next, adaptors were ligated to the 5 -end of the first-strand cDNA by DNA ligase (TaKaRa, Dalian, China), and the second-strand cDNA was synthesized through primer extension using Ex Taq polymerase (TaKaRa, Dalian, China). Last, the polyA tail and 5' adaptors were removed by GsuI digestion. The synthesized cDNA was fractioned ultrasonically into 300-800 bp fragments and purified using Ampure beads (Agencourt, America). The prepared cDNAs were transformed into libraries with the TruSeq DNA Sample Prep Kit Set A (Illumina, America), clonally amplified using the TruSeq PE Cluster Kit (Illumina, America) and sequenced with Illumina equipment according to the manufacturer's instructions. Clean reads (those in which at least half of the bases, excluding $\mathrm{N}$, had a quality score above 5) were retained and mapped onto the genome or annotated genes of strain Z5. Reads were collected from the different samples for each gene, and read counts were transformed into RPM values (Reads Per Kilo bases per Million reads) [71]. Using the MA-plot-based method with Random Sampling model in the DEGseq program package [72], the differences in expression levels between samples were calculated for each individual gene. Differences were determined to be significant if FDR scores were $\leq 0.001$.

\section{Gene expression analysis}

The genes with significantly different transcriptional levels in all 4 transcriptomes need to have RPM (Reads Per Kilo bases per Million reads) scores above 10 in all transcriptomes as well as the coefficient of variation of the RPM values in all transcriptomes of not less than 0.6. The results were organized and visualized using Pearson correlations and hierarchical clustering with the average linkage clustering method to view the whole data set. K-means cluster analysis was used to group the genes into 10 clusters with the TIGR multiexperiment viewer (MeV, http://www.tigr.org/software). For the purposes of GO terms enrichment analysis, genes that differed significantly between treatment (XGEP, CGEP or RGEP) and control (SGEP) were those that had $R P M \geq 10$, q-value $\leq 0.0001$ and $\mid \log _{2}$ of the ratio of the RPM values $\mid \geq 2$. GO terms annotation was accomplished by Blast2GO software (http://www.blast2go.com). Genes that differed significantly between treatment and control were analyzed and visualized to see the results of GO term enrichment using the classic algorithm and Fisher's exact test in the Bioconductor topGO package (http:// www.bioconductor.org/packages/2.12/bioc/html/topGO. $\mathrm{html}$ ). Detrended correspondence analysis (DCA) was conducted using the vegan package of $R$ [73]. The transcriptome sequencing data and screening results are provided in Additional file 17.

\section{Protein classification}

Whole genome protein families were classified by InterproScan analysis (http://www.ebi.ac.uk/interpro/). The number of genes for each COG category was determined using a BlastP search against the COG data set. The highest scoring hit to COG category for each protein was retained. Using the same way, the sugar transporter family proteins were found out by blasting to Transporter Classification Database (TCDB, http://www.tcdb.org/). CAZyme proteins were classified with the CAZYmes Analysis Toolkit [74] (CAT, http://mothra.ornl.gov/cgi-bin/cat.cgi). Annotation based on sequence similarity and annotation based on association rules between CAZy families and pfam domains against the CAZy database (http://www.cazy.org/) were integrated for CAZyme protein predictions.

\section{Gene cluster identification}

The gene families were collected by visual inspection using CIRCOS software [75] (http://www.circos.ca). For CAZyme gene clusters, a cluster is defined as a region containing a statistically higher proportion of a particular gene family, and the cluster must begin and end with a gene from the family studied. We then calculated the probability of these CAZyme gene clusters based on a hypergeometric distribution, and all 44 clusters had a $P$ value $<0.01$. To analyze fungal clusters of secondary metabolism, the genome annotation data were coordinated and analyzed with the program SMURF (http:// www.jcvi.org/smurf/index.php). The nucleotide fragments in which genes were nearly co-regulated (up- or down-) were also collected by visual inspection and compared to the clusters of secondary metabolism by Blastp.

\section{Accession number}

This Whole Genome Shotgun project has been deposited at DDBJ/EMBL/GenBank under the accession AZZA 
00000000. The version described in this paper is version AZZA01000000. The transcriptomes data discussed in this publication have been deposited in NCBI's Gene Expression Omnibus [76] and are accessible through GEO Series accession number GSE55086 (http://www.ncbi. nlm.nih.gov/geo/query/acc.cgi?acc=GSE55086).

\section{Additional files}

Additional file 1: Phylogenetic analysis of strain Z5 in Aspergillus section Fumigati. Phylogenetic analysis of strain $\mathrm{Z} 5$ was performed in Aspergillus section Fumigati based on the $\beta$-tubulin, calmodulin and ITS sequences

Additional file 2: Scaffolds matching. All Z5 scaffolds were matched into the chromosomes of A. fumigatus AF293.

Additional file 3: Comparison of protein domains in six filamentous fungi. The numbers of each protein domain identified by InterPro were compared within A. fumigatus, A. nidulans, A. niger, A. oryzae, T. reesei and N. crassa.

Additional file 4: Plant biomass-related protein families in A. fumigatus Z5. Plant biomass-related protein families were found out in the six filamentous fungi and $\mathrm{S}$. cerevisiae.

Additional file 5: Comparison of gene numbers for each COG category. The predicted numbers of each COG category for seven fungi including A. fumigatus $Z 5$ were listed.

Additional file 6: CAZyme genes. All the predicted CAZyme genes in A. fumigatus $Z 5$ were listed.

Additional file 7: Plant biomass-degrading enzymes in A. fumigatus Z5. Cellulase genes, hemicellulase genes and pectinase genes were found out from the genome of Z5, and the properties (protein family, signal peptide, secretome signal and homologs in other fungi) of these enzymes were also investigated.

Additional file 8: CAZyme gene clusters in A. fumigatus Z5. 44 CAZyme gene clusters were found in Z5 genome, and p-values $<0.01$.

Additional file 9: Maps of scaffolds. The map of scaffold 2 was shown by Fig. 4 with the detailed descriptions, and this file contained the maps for the remained scaffolds.

Additional file 10: Differential expression of secretory pathway genes. This file shown the expression values of genes in secretory pathway in A. fumigatus $Z 5$ when induced by different carbon source.

Additional file 11: Homologs among the rice straw-induced strain Z5 and wheat straw-induced $A$. niger and $T$. reesei. This file listed the homologs of genes that were differently expressed in rice straw-induced Z5, wheat straw-induced A. niger and T. reesei.

Additional file 12: GO enrichment analysis. 444, 1386 and 1711 significantly differently expressed genes (xylan to sucrose, rice straw to sucrose and cellulose to sucrose, respectively) were used to perform GO enrichment analysis. The results were shown in this file.

Additional file 13: Co-regulated fragments in $\mathbf{Z 5}$ genome. 35 co-regulated fragments consisting of continuous genes in genome were found and listed in this file.

Additional file 14: Secondary metabolite. 30 secondary metabolite biosynthetic gene clusters and related PKS genes NPRS genes in Z5 genome were shown in this file.

Additional file 15: Co-regulated genes in Aspergillus genus when grown on xylose. 23 co-regulated genes in Aspergillus genus identified by Andersen et al. when grown on xylose rather than glucose were also conserved in transcriptome data in this study.

Additional file 16: The primers for qRT-PCR experiments. The primers of 53 genes were listed in this file.
Additional file 17: Transcriptome screening results. This file contained the selected genes for the analysis of Pearson correlations and hierarchical clustering and $\mathrm{GO}$ enrichment.

\section{Competing interests}

The authors declare that they have no competing interests.

\section{Authors' contributions}

YM performed the majority of the experiments and wrote the manuscript DL performed the DNA and RNA extraction and assisted in the drafting and revision of the manuscript. GL and PL performed the strain incubation. YX and QS were involved in the data analysis. RZ designed and supervised the experiments and contributed to the manuscript writing and revision. All authors read and approved the final manuscript.

\section{Acknowledgements}

This research was financially supported by the Chinese Ministry of Science and Technology (2013AA102802) and the Chinese Ministry of Agriculture (2011-G27). DL was supported by the National Natural Science Foundation of China for Youth (31201685), and RZ and QS were also supported by the 111 Project (B12009) and the Priority Academic Program Development (PAPD) of Jiangsu Higher Education Institutions respectively. The authors thank Irina Druzhinina and Christian P. Kubicek of Vienna University of Technology

(Austria) for their critical review of this manuscript.

Received: 19 March 2014 Accepted: 28 May 2015

Published online: 16 June 2015

\section{References}

1. Goldemberg J. Ethanol for a Sustainable Energy Future. Science. 2007:315:808-10

2. Sticklen MB. Plant genetic engineering for biofuel production: towards affordable cellulosic ethanol. Nat Rev Genet. 2008;9:433-43.

3. Himmel ME, Ding S-Y, Johnson DK, Adney WS, Nimlos MR, Brady JW, Foust TD. Biomass recalcitrance: engineering plants and enzymes for biofuels production. Science. 2007;315:804-7.

4. Lynd LR, Wyman CE, Gerngross TU. Biocommodity Engineering. Biotechnol Prog. 1999;15:777-93.

5. Horn SJ, Vaaje-Kolstad G, Westereng B, Eijsink VG. Novel enzymes for the degradation of cellulose. Biotechnol Biofuels. 2012:5:1-13.

6. Wilson DB. Three Microbial Strategies for Plant Cell Wall Degradation. Ann N Y Acad Sci. 2008:1125:289-97.

7. Himmel ME, Bayer EA. Lignocellulose conversion to biofuels: current challenges, global perspectives. Curr Opin Biotechnol. 2009;20:316-7.

8. Patel-Predd P. Overcoming the hurdles to producing ethanol from cellulose. Environ Sci Technol. 2006:40:4052-3

9. Bloom JD, Meyer MM, Meinhold P, Otey CR, MacMillan D, Arnold FH. Evolving strategies for enzyme engineering. Curr Opin Struct Biol. 2005:15:447-52

10. Kaper T, Brouns S, GEERLING A, De Vos W, Van der Oost J. DNA family shuffling of hyperthermostable $\beta$-glycosidases. Biochem J. 2002;368:461-70.

11. Boer $\mathrm{H}$, Koivula A. The relationship between thermal stability and $\mathrm{pH}$ optimum studied with wild-type and mutant Trichoderma reesei cellobiohydrolase Cel7A. Eur J Biochem. 2003;270:841-8.

12. Percival Zhang Y-H, Himmel ME, Mielenz JR. Outlook for cellulase improvement: Screening and selection strategies. Biotechnol Adv. 2006:24:452-81.

13. Aro N, Pakula T, Penttilä M. Transcriptional regulation of plant cell wall degradation by filamentous fungi. FEMS Microbiol Rev. 2005;29:719-39.

14. Punt PJ, van Biezen N, Conesa A, Albers A, Mangnus J, van den Hondel C. Filamentous fungi as cell factories for heterologous protein production. Trends Biotechnol. 2002:20:200-6.

15. Borkovich KA, Alex LA, Yarden O, Freitag M, Turner GE, Read ND, Seiler $S$, Bell-Pedersen D, Paietta J, Plesofsky N. Lessons from the genome sequence of Neurospora crassa: tracing the path from genomic blueprint to multicellular organism. Microbiol Mol Biol Rev. 2004;68:1-108.

16. Galagan JE, Calvo SE, Borkovich KA, Selker EU, Read ND, Jaffe D, FitzHugh W, Ma L-J, Smirnov S, Purcell $S$. The genome sequence of the filamentous fungus Neurospora crassa. Nature. 2003;422:859-68. 
17. Colot HV, Park G, Turner GE, Ringelberg C, Crew CM, Litvinkova L, Weiss RL, Borkovich KA, Dunlap JC. A high-throughput gene knockout procedure for Neurospora reveals functions for multiple transcription factors. Proc Natl Acad Sci U S A. 2006;103:10352-7.

18. Nevalainen KMH, Te'o VSJ, Bergquist PL. Heterologous protein expression in filamentous fungi. Trends Biotechnol. 2005;23:468-74.

19. Van den Hondel CA, Punt PJ, van Gorcom RF. Production of extracellular proteins by the filamentous fungus Aspergillus. Antonie Van Leeuwenhoek. 1992;61:153-60.

20. Cherry JR, Fidantsef AL. Directed evolution of industrial enzymes: an update. Curr Opin Biotechnol. 2003;14:438-43.

21. Tian C, Beeson WT, lavarone AT, Sun J, Marletta MA, Cate JHD, Glass NL. Systems analysis of plant cell wall degradation by the model filamentous fungus Neurospora crassa. Proc Natl Acad Sci. 2009;106:22157-62.

22. Delmas S, Pullan ST, Gaddipati S, Kokolski M, Malla S, Blythe MJ, Ibbett R, Campbell M, Liddell S, Aboobaker A. Uncovering the genome-wide transcriptional responses of the filamentous fungus Aspergillus niger to lignocellulose using RNA sequencing. PLoS Genet. 2012;8, e1002875.

23. Meyer AS, Rosgaard L, Sørensen HR. The minimal enzyme cocktail concept for biomass processing. J Cereal Sci. 2009;50:337-44.

24. Chen X, Ishida N, Todaka N, Nakamura R, Maruyama J, Takahashi H, Kitamoto K. Promotion of efficient saccharification of crystalline cellulose by Aspergillus fumigatus Swo1. Appl Environ Microbiol. 2010;76:2556-61.

25. Liu D, Li J, Zhao S, Zhang R, Wang M, Miao Y, Shen Y, Shen Q. Secretome diversity and quantitative analysis of cellulolytic Aspergillus fumigatus Z5 in the presence of different carbon sources. Biotechnol Biofuels. 2013;6:149.

26. Liu D, Zhang R, Yang X, Wu H, Xu D, Tang Z, Shen Q. Thermostable cellulase production of Aspergillus fumigatus Z5 under solid-state fermentation and its application in degradation of agricultural wastes. Int Biodeterior Biodegrad. 2011;65:717-25.

27. Samson RA, Hong S, Peterson SW, Frisvad JC, Varga J. Polyphasic taxonomy of Aspergillus section Fumigati and its teleomorph Neosartorya. Stud Mycol. 2007;59:147-203

28. Ansorge WJ. Next-generation DNA sequencing techniques. New Biotechnol. 2009;25:195-203.

29. Nierman WC, Pain A, Anderson MJ, Wortman JR, Kim HS, Arroyo J, Berriman M, Abe K, Archer DB, Bermejo C. Genomic sequence of the pathogenic and allergenic filamentous fungus Aspergillus fumigatus. Nature. 2005;438:1151-6.

30. Zdobnov EM, Apweiler R. InterProScan-an integration platform for the signature-recognition methods in InterPro. Bioinformatics. 2001;17:847-8.

31. Machida M, Asai K, Sano M, Tanaka T, Kumagai T, Terai G, Kusumoto K-I, Arima T, Akita O, Kashiwagi Y. Genome sequencing and analysis of Aspergillus oryzae. Nature. 2005;438:1157-61.

32. Tatusov RL, Fedorova ND, Jackson JD, Jacobs AR, Kiryutin B, Koonin EV, Krylov DM, Mazumder R, Mekhedov SL, Nikolskaya AN. The COG database: an updated version includes eukaryotes. BMC Bioinformatics. 2003;4:41.

33. Cantarel BL, Coutinho PM, Rancurel C, Bernard T, Lombard V, Henrissat B. The Carbohydrate-Active EnZymes database (CAZy): an expert resource for glycogenomics. Nucleic Acids Res. 2009;37 suppl 1:D233-8.

34. Zhao Z, Liu H, Wang C, Xu J-R. Comparative analysis of fungal genomes reveals different plant cell wall degrading capacity in fungi. BMC Genomics. 2013;14:274

35. Rapoport TA. Protein translocation across the eukaryotic endoplasmic reticulum and bacterial plasma membranes. Nature. 2007:450:663-9.

36. Pel HJ, De Winde JH, Archer DB, Dyer PS, Hofmann G, Schaap PJ, Turner G, de Vries RP, Albang R, Albermann K. Genome sequencing and analysis of the versatile cell factory Aspergillus niger CBS 513.88. Nat Biotechnol. 2007;25:221-31.

37. Schröder M, Kaufman RJ. ER stress and the unfolded protein response. Mutat Res Mol Mech Mutagen. 2005;569:29-63.

38. Travers KJ, Patil CK, Wodicka L, Lockhart DJ, Weissman JS, Walter P. Functional and genomic analyses reveal an essential coordination between the unfolded protein response and ER-associated degradation. Cell. 2000;101:249-58.

39. Van Huizen R, Martindale $\mathrm{L}$, Gorospe M, Holbrook NJ. P58IPK, a novel endoplasmic reticulum stress-inducible protein and potential negative regulator of elF2a signaling. J Biol Chem. 2003;278:15558-64.

40. Martinez D, Berka RM, Henrissat B, Saloheimo M, Arvas M, Baker SE, Chapman J, Chertkov O, Coutinho PM, Cullen D. Genome sequencing and analysis of the biomass-degrading fungus Trichoderma reesei (syn. Hypocrea jecorina). Nat Biotechnol. 2008;26:553-60.
41. Mach-Aigner AR, Pucher ME, Mach RL. D-Xylose as a repressor or inducer of xylanase expression in Hypocrea jecorina (Trichoderma reesei). Appl Environ Microbiol. 2010;76:1770-6.

42. Prathumpai W, Mcintyre M, Nielsen J. The effect of CreA in glucose and xylose catabolism in Aspergillus nidulans. Appl Microbiol Biotechnol. 2004;63:748-53.

43. Phillips CM, Beeson IV WT, Cate JH, Marletta MA. Cellobiose dehydrogenase and a copper-dependent polysaccharide monooxygenase potentiate cellulose degradation by Neurospora crassa. ACS Chem Biol. 2011;6:1399-406.

44. Saier MH. Families of transmembrane sugar transport proteins. Mol Microbiol. 2000;35:699-710.

45. Ivanova C, Baath JA, Seiboth B, Kubicek CP. Systems analysis of lactose metabolism in Trichoderma reesei identifies a lactose permease that is essential for cellulase induction. PLoS One. 2013;8, e62631.

46. Ries L, Pullan ST, Delmas S, Malla S, Blythe MJ, Archer DB. Genome-wide transcriptional response of Trichoderma reesei to lignocellulose using RNA sequencing and comparison with Aspergillus niger. BMC Genomics. 2013;14:541.

47. Alexa A. Rahnenfuhrer J: topGO: enrichment analysis for gene ontology. R Package Version. 2010;2.

48. Pontercorvo G, Roper JA, Hemmons LM, Macdonald KD, Bufton AWJ. The genetics of Aspergillus nidulans. Adv Genet. 1953;5:141-238.

49. Fedorova ND, Khaldi N, Joardar VS, Maiti R, Amedeo P, Anderson MJ, Crabtree J, Silva JC, Badger JH, Albarraq A. Genomic islands in the pathogenic filamentous fungus Aspergillus fumigatus. PLoS Genet. 2008;4, e1000046.

50. Liu D, Zhang R, Yang X, Zhang Z, Song S, Miao Y, Shen Q. Characterization of a thermostable $\beta$-glucosidase from Aspergillus fumigatus Z5, and its functional expression in Pichia pastoris X33. Microb Cell Factories. 2012;11:25.

51. Ximenes EA, Felix CR, Ulhoa CJ. Production of cellulases by Aspergillus fumigatus and characterization of one $\beta$-glucosidase. Curr Microbiol. 1996;32:119-23.

52. Rementeria A, López-Molina N, Ludwig A, Vivanco AB, Bikandi J, Pontón J, Garaizar J. Genes and molecules involved in Aspergillus fumigatus virulence. Rev Iberoam Micol. 2005;22:1-23.

53. Jørgensen T, Goosen T, van den Hondel C, Ram A, Iversen J. Transcriptomic comparison of Aspergillus niger growing on two different sugars reveals coordinated regulation of the secretory pathway. BMC Genomics. 2009;10:44.

54. Guillemette T, van Peij NN, Goosen T, Lanthaler K, Robson GD, van den Hondel CA, Stam H, Archer DB. Genomic analysis of the secretion stress response in the enzyme-producing cell factory Aspergillus niger. BMC Genomics. 2007;8:158.

55. Deshpande N, Wilkins MR, Packer N, Nevalainen H. Protein glycosylation pathways in filamentous fungi. Glycobiology. 2008;18:626-37.

56. Van Peij NN, Visser J, De Graaff LH. Isolation and analysis of XInR, encoding a transcriptional activator co-ordinating xylanolytic expression in Aspergillus niger. Mol Microbiol. 1998;27:131-42.

57. De Groot MJ, van den Dool C, Wösten HA, Levisson M, VanKuyk PA, Ruijter GJ, de Vries RP. Regulation of pentose catabolic pathway genes of Aspergillus niger. Food Technol Biotechnol. 2007;45:134-8.

58. Hasper AA, Visser J, De Graaff LH. The Aspergillus niger transcriptional activator XInR, which is involved in the degradation of the polysaccharides xylan and cellulose, also regulates $d$-xylose reductase gene expression. Mol Microbiol. 2000;36:193-200

59. Gielkens MM, Dekkers E, Visser J, de Graaff LH. Two cellobiohydrolaseencoding genes from Aspergillus niger require D-xylose and the xylanolytic transcriptional activator XInR for their expression. Appl Environ Microbiol. 1999:65:4340-5.

60. Andersen MR, Vongsangnak W, Panagiotou G, Salazar MP, Lehmann L, Nielsen J. A trispecies Aspergillus microarray: Comparative transcriptomics of three Aspergillus species. Proc Natl Acad Sci. 2008;105:4387-92.

61. Znameroski EA, Coradetti ST, Roche CM, Tsai JC, lavarone AT, Cate JH, Glass NL. Induction of lignocellulose-degrading enzymes in Neurospora crassa by cellodextrins. Proc Natl Acad Sci. 2012;109:6012-7.

62. Dowzer CE, Kelly JM. Analysis of the creA gene, a regulator of carbon catabolite repression in Aspergillus nidulans. Mol Cell Biol. 1991;11:5701-9.

63. Nehlin JO, Ronne H. Yeast MIG1 repressor is related to the mammalian early growth response and Wilms' tumour finger proteins. EMBO J. 1990;9:2891.

64. Ruijter GJ, Vanhanen SA, Gielkens MM, van de Vondervoort PJ, Visser J. Isolation of Aspergillus niger creA mutants and effects of the mutations on expression of arabinases and L-arabinose catabolic enzymes. Microbiology. 1997;143:2991-8. 
65. Schatz MC, Delcher AL, Salzberg SL. Assembly of large genomes using second-generation sequencing. Genome Res. 2010;20:1165-73.

66. Kim P-G, Cho H-G, Park K. A scaffold analysis tool using mate-pair information in genome sequencing. BioMed Res Int 2008. 2008.

67. Lowe TM, Eddy SR. tRNAscan-SE: a program for improved detection of transfer RNA genes in genomic sequence. Nucleic Acids Res. 1997;25:0955-964.

68. Petersen TN, Brunak S, von Heijne G, Nielsen H. SignalP 4.0: discriminating signal peptides from transmembrane regions. Nat Methods. 2011;8:785-6.

69. Hart TD, De Leij F, Kinsey G, Kelley J, Lynch JM. Strategies for the isolation of cellulolytic fungi for composting of wheat straw. World J Microbiol Biotechnol. 2002;18:471-80

70. Ng P, Wei C-L, Sung W-K, Chiu KP, Lipovich L, Ang CC, Gupta S, Shahab A, Ridwan A, Wong CH. Gene identification signature (GIS) analysis for transcriptome characterization and genome annotation. Nat Methods. 2005;2:105-11.

71. Mortazavi A, Williams BA, McCue K, Schaeffer L, Wold B. Mapping and quantifying mammalian transcriptomes by RNA-Seq. Nat Methods. 2008;:5:621-8.

72. Wang L, Feng Z, Wang X, Wang X, Zhang X. DEGseq: an R package for identifying differentially expressed genes from RNA-seq data. Bioinformatics. 2010:26:136-8.

73. Oksanen J, Blanchet FG, Kindt R, Legendre P, Minchin PR, others: Vegan: Community Ecology Package. R Package Version 1: R Package Version 2.0-6. 2013.

74. Park BH, Karpinets TV, Syed MH, Leuze MR, Uberbacher EC. CAZymes Analysis Toolkit (CAT): web service for searching and analyzing carbohydrate-active enzymes in a newly sequenced organism using CAZy database. Glycobiology. 2010;20:1574-84.

75. Krzywinski M, Schein J, Birol I, Connors J, Gascoyne R, Horsman D, Jones SJ, Marra MA. Circos: an information aesthetic for comparative genomics. Genome Res. 2009:19:1639-45.

76. Edgar R, Domrachev M, Lash AE. Gene Expression Omnibus: NCBI gene expression and hybridization array data repository. Nucleic Acids Res. 2002;30:207-10.

\section{Submit your next manuscript to BioMed Central and take full advantage of:}

- Convenient online submission

- Thorough peer review

- No space constraints or color figure charges

- Immediate publication on acceptance

- Inclusion in PubMed, CAS, Scopus and Google Scholar

- Research which is freely available for redistribution 